\title{
On Partial Contraction Analysis for Coupled Nonlinear Oscillators
}

\author{
Wei Wang and Jean-Jacques E. Slotine \\ Nonlinear Systems Laboratory \\ Massachusetts Institute of Technology \\ Cambridge, Massachusetts, 02139, USA \\ wangwei@mit.edu, jjs@mit.edu
}

\begin{abstract}
We describe a simple but general method to analyze networks of coupled identical nonlinear oscillators, and study applications to fast synchronization, locomotion, and schooling. Specifically, we use nonlinear contraction theory to derive exact and global (rather than linearized) results on synchronization, anti-synchronization and oscillator-death. The method can be applied to coupled networks of various structures and arbitrary size. For oscillators with positive-definite diffusion coupling, it can be shown that synchronization always occur globally for strong enough coupling strengths, and an explicit upper bound on the corresponding threshold can be computed through eigenvalue analysis. The discussion also extends to the case when network structure varies abruptly and asynchronously, as in "flocks" of oscillators or dynamic elements.
\end{abstract}

\section{Introduction}

Initiated by Huygens in the 17th century, the study of coupled oscillators involves today a variety of research fields, such as mathematics $[13,58,74,75]$, biology [10, 48, 73], neuroscience [6, 24, $31,43,50,64,87,88]$, robotics [4, 32], and electronics [9], to cite just a few. Theoretical analysis of coupled oscillators can be performed either in phase-space, as e.g. in the classical Kuramoto model [33, 75, 85], or in state-space, as e.g. in the fast threshold modulation model [30, 70, 71]. While nonlinear state-space models are much closer to physical reality and apply to arbitrary initial conditions, there still does not exist a general and explicit analysis tool to study them. In this paper, a new method is developed based on contraction analysis to study dynamic behaviors of coupled nonlinear oscillators, with an emphasis on the study of spontaneous synchronization.

Basically, a nonlinear dynamic system is called contracting if initial conditions or temporary disturbances are forgotten exponentially fast, so that all trajectories converge to a unique trajectory. After a brief review of key results of contraction theory [40, 41, 39] in Section 2, we introduce the new concept of partial contraction, which extends contraction analysis to include convergence to behaviors or to specific properties (such as equality of state components, or convergence to a manifold). Partial contraction provides a very general analysis tool to investigate the stability of large-scale systems. It is especially powerful to study synchronization behaviors, and it inherits from contraction the feature that convergence and long-term behavior can be analyzed separately, leading to significant conceptual simplifications.

Section 3 first illustrates the method by analyzing the synchronization behaviors of two coupled oscillators. Section 4 then generalizes the analysis to coupled networks of various structures and arbitrary size. For nonlinear systems with positive-definite diffusive couplings, synchronization will always occur if coupling strengths are strong enough, and an explicit upper bound on the corresponding threshold can be computed through eigenvalue analysis. The results are exact and 
global, rather than linearized, and can be easily extended to study nonlinear couplings, unidirectional couplings, and positive semi-definite couplings. We relate the synchronization rate to the network's geometric properties, such as connectivity, graph diameter or mean distance. A fast inhibition mechanism is studied. Finally, we build flocking and schooling models by extending the previous analysis to coupled networks with switching topology, and also build the network models with a leader-followers structure. Concluding remarks are offered in Section 5.

Most of the results in the paper are illustrated using Van der Pol oscillators, whose relaxation behavior can be made to resemble closely some standard neuron models, for instance. In contrast with previous approaches such as e.g., $[8,57,72]$, the results are exact and global.

\section{Contraction and Partial Contraction}

Basically, a nonlinear time-varying dynamic system will be called contracting if initial conditions or temporary disturbances are forgotten exponentially fast, i.e., if trajectories of the perturbed system return to their nominal behavior with an exponential convergence rate. The concept of partial contraction allows one to extend the applications of contraction analysis to include convergence to behaviors or to specific properties (such as equality of state components, or convergence to a manifold) rather than trajectories.

\subsection{Contraction Theory}

We briefly summarize the basic definitions and main results of Contraction Theory here, details of which can be found in [40]. Consider a nonlinear system

$$
\dot{\mathbf{x}}=\mathbf{f}(\mathbf{x}, t)
$$

where $\mathbf{x} \in \mathbb{R}^{m \times 1}$ is a state vector and $\mathbf{f}$ is an $m \times 1$ vector function. Assuming $\mathbf{f}(\mathbf{x}, t)$ is continuously differentiable, we have

$$
\frac{d}{d t}\left(\delta \mathbf{x}^{T} \delta \mathbf{x}\right)=2 \delta \mathbf{x}^{T} \delta \dot{\mathbf{x}}=2 \delta \mathbf{x}^{T} \frac{\partial \mathbf{f}}{\partial \mathbf{x}} \delta \mathbf{x} \leq 2 \lambda_{\max } \delta \mathbf{x}^{T} \delta \mathbf{x}
$$

where $\delta \mathbf{x}$ is a virtual displacement between two neighboring solution trajectories, and $\lambda_{\max }(\mathbf{x}, t)$ is the largest eigenvalue of the symmetric part of the Jacobian $\mathbf{J}=\frac{\partial \mathbf{f}}{\partial \mathbf{x}}$. Hence, if $\lambda_{\max }(\mathbf{x}, t)$ is uniformly strictly negative, any infinitesimal length $\|\delta \mathbf{x}\|$ converges exponentially to zero. By path integration at fixed time, this implies in turn that all the solutions of the system (1) converge exponentially to a single trajectory, independently of the initial conditions.

More generally, consider a coordinate transformation

$$
\delta \mathbf{z}=\boldsymbol{\Theta} \delta \mathbf{x}
$$

where $\boldsymbol{\Theta}(\mathbf{x}, t)$ is a uniformly invertible square matrix. One has

$$
\frac{d}{d t}\left(\delta \mathbf{z}^{T} \delta \mathbf{z}\right)=2 \delta \mathbf{z}^{T} \delta \dot{\mathbf{z}}=2 \delta \mathbf{z}^{T}\left(\dot{\boldsymbol{\Theta}}+\boldsymbol{\Theta} \frac{\partial \mathbf{f}}{\partial \mathbf{x}}\right) \Theta^{-1} \delta \mathbf{z}
$$

so that exponential convergence of $\|\delta \mathbf{z}\|$ to zero is guaranteed if the generalized Jacobian matrix

$$
\mathbf{F}=\left(\dot{\boldsymbol{\Theta}}+\boldsymbol{\Theta} \frac{\partial \mathbf{f}}{\partial \mathbf{x}}\right) \boldsymbol{\Theta}^{-1}
$$

is uniformly negative definite. Again, this implies in turn that all the solutions of the original system (1) converge exponentially to a single trajectory, independently of the initial conditions. 
By convention, the system (1) is called contracting, $\mathbf{f}(\mathbf{x}, t)$ is called a contracting function, and the absolute value of the largest eigenvalue of the symmetric part of $\mathbf{F}$ is called the system's contraction rate with respect to the uniformly positive definite metric $\mathbf{M}=\mathbf{\Theta}^{T} \boldsymbol{\Theta}$. Note that in a globally contracting autonomous system, all trajectories converge exponentially to a unique equilibrium point $[40,67]$.

\subsection{Feedback Combination of Contracting Systems}

One of the main features of contraction is that it is automatically preserved through a variety of system combinations. Here we extend the study of feedback combination in [40] and derive a result we will use in section 4.4. Consider two contracting systems and an arbitrary feedback connection between them. The overall dynamics of the generalized virtual displacements $\delta \mathbf{z}_{i}$ can be written as

$$
\frac{d}{d t}\left[\begin{array}{l}
\delta \mathbf{z}_{1} \\
\delta \mathbf{z}_{2}
\end{array}\right]=\mathbf{F}\left[\begin{array}{l}
\delta \mathbf{z}_{1} \\
\delta \mathbf{z}_{2}
\end{array}\right]
$$

with the symmetric part of the generalized Jacobian in the form

$$
\mathbf{F}_{s}=\frac{1}{2}\left(\mathbf{F}+\mathbf{F}^{T}\right)=\left[\begin{array}{cc}
\mathbf{F}_{1 s} & \mathbf{G} \\
\mathbf{G}^{T} & \mathbf{F}_{2 s}
\end{array}\right]
$$

where the subscript " $s$ " refers to the symmetric part of a matrix. By hypothesis the matrices $\mathbf{F}_{1 s}$ and $\mathbf{F}_{2 s}$ are uniformly negative definite. Thus $\mathbf{F}$ is uniformly negative definite if and only if ([25], page 472 )

$$
\mathbf{F}_{2 s}<\mathbf{G}^{T} \mathbf{F}_{1 s}^{-1} \mathbf{G}
$$

Thus, a sufficient condition for the overall system to be contracting is that

$$
\lambda\left(\mathbf{F}_{1 s}\right) \lambda\left(\mathbf{F}_{2 s}\right)>\sigma^{2}(\mathbf{G}) \quad \text { uniformly } \forall \mathrm{t} \geq 0
$$

where $\lambda\left(\mathbf{F}_{i s}\right)$ is the contraction rate of $\mathbf{F}_{i s}$ and $\sigma(\mathbf{G})$ is the largest singular value of $\mathbf{G}$. Indeed, condition (2) is equivalent to

$$
\lambda_{\max }\left(\mathbf{F}_{2 s}\right)<\lambda_{\min }\left(\mathbf{F}_{1 s}^{-1}\right) \sigma^{2}(\mathbf{G})
$$

and, for an arbitrary nonzero vector $\mathbf{v}$,

$$
\mathbf{v}^{T} \mathbf{F}_{2 s} \mathbf{v}<\lambda_{\min }\left(\mathbf{F}_{1 s}^{-1}\right) \sigma^{2}(\mathbf{G}) \mathbf{v}^{T} \mathbf{v} \leq \mathbf{v}^{T} \mathbf{G}^{T} \mathbf{F}_{1 s}^{-1} \mathbf{G} \mathbf{v}
$$

The result can be applied recursively to larger combinations. Note that, from the eigenvalue interlacing theorem [25],

$$
\lambda\left(\mathbf{F}_{s}\right) \leq \min _{i} \lambda\left(\mathbf{F}_{i s}\right) .
$$

\subsection{Partial Contraction}

We now introduce the concept of partial contraction, which forms the basis of this paper. It derives from the very simple but very general result which follows.

Theorem 1 Consider a nonlinear system of the form

$$
\dot{\mathbf{x}}=\mathbf{f}(\mathbf{x}, \mathbf{x}, t)
$$


and assume that the auxiliary system

$$
\dot{\mathbf{y}}=\mathbf{f}(\mathbf{y}, \mathbf{x}, t)
$$

is contracting with respect to $\mathbf{y}$. If a particular solution of the auxiliary $\mathbf{y}$-system verifies a smooth specific property, then all trajectories of the original $\mathbf{x}$-system verify this property exponentially. The original system is said to be partially contracting.

Proof: The virtual, observer-like $\mathbf{y}$-system has two particular solutions, namely $\mathbf{y}(t)=\mathbf{x}(t)$ for all $t \geq 0$ and the solution with the specific property. Since all trajectories of the $\mathbf{y}$-system converge exponentially to a single trajectory, this implies that $\mathbf{x}(t)$ verifies the specific property exponentially.

Example 2.1: Consider a system of the form

$$
\dot{\mathbf{x}}=\mathbf{c}(\mathbf{x}, t)+\mathbf{d}(\mathbf{x}, t)
$$

where function $\mathbf{c}$ is contracting in a constant metric ${ }^{1}$. The auxiliary contracting system may then be constructed as

$$
\dot{\mathbf{y}}=\mathbf{c}(\mathbf{y}, t)+\mathbf{d}(\mathbf{x}, t)
$$

The specific property may consist e.g. a relationship between state variables, or simply of a particular trajectory.

Example 2.2: Consider a convex combination or interpolation between contracting dynamics

$$
\dot{\mathbf{x}}=\sum_{i} \alpha_{i}(\mathbf{x}, t) \mathbf{f}_{i}(\mathbf{x}, t)
$$

where the individual systems $\dot{\mathbf{x}}=\mathbf{f}_{i}(\mathbf{x}, t)$ are contracting in a common metric $\mathbf{M}(\mathbf{x})=\Theta^{T}(\mathbf{x}) \Theta(\mathbf{x})$ and have a common trajectory $\mathbf{x}_{o}(t)$ (for instance an equilibrium), with all $\alpha_{i}(\mathbf{x}, t) \geq 0$ and $\sum_{i} \alpha_{i}(\mathbf{x}, t)=1$. Then all trajectories of the system globally exponentially converge to the trajectory $\mathbf{x}_{o}(t)$. Indeed, the auxiliary system

$$
\dot{\mathbf{y}}=\sum_{i} \alpha_{i}(\mathbf{x}, t) \mathbf{f}_{i}(\mathbf{y}, t)
$$

is contracting (with metric $\mathbf{M}(\mathbf{y})$ ) and has $\mathbf{x}(t)$ and $\mathbf{x}_{o}(t)$ as particular solutions.

Example 2.3: Recent research in computational neuroscience points out the importance of continuous attractors [63, 35]. Consider [22] a nonlinear neural network model

$$
\tau \dot{x}_{i}+x_{i}=\left[\sum_{j} \mathbf{w}_{j i} x_{j}+b_{i}\right]^{+} \quad i=1, \ldots, n
$$

with $[a]^{+}=\max (0, a)$ and constant $\tau>0$, or in matrix form

$$
\tau \dot{\mathbf{x}}+\mathbf{x}=[\mathbf{W} \mathbf{x}+\mathbf{b}]^{+}
$$

with $\mathbf{W}^{T}=\mathbf{W}=\left[\mathbf{w}_{i j}\right]$. If $\mathbf{I}-\mathbf{W}$ is positive semi-definite and $\mathbf{b}$ is in its range space, a line attractor exists [22]. To prove global exponential stability of this line attractor, arrange the eigenvalues $\lambda_{i}$ of $\mathbf{I}-\mathbf{W}$ in increasing order, with $\lambda_{2}>\lambda_{1}=0$. The corresponding eigenvectors $\mathbf{u}_{i}$ represent an orthonormal basis of the state space. Consider now the auxiliary system

$$
\tau \dot{\mathbf{y}}+\mathbf{y}=[\mathbf{W} \mathbf{y}+\mathbf{b}]^{+}+\lambda_{2} \mathbf{u}_{1} \mathbf{u}_{1}^{T}(\mathbf{x}(t)-\mathbf{y})
$$

Note that given positive initial conditions, all components of $\mathbf{x}$ and $\mathbf{y}$ remain positive. The $\mathbf{y}$-system can be shown to be contracting with rate $\lambda_{2} / 2 \tau$ [82] using contraction analysis results for continuously

\footnotetext{
${ }^{1}$ This condition of a constant metric is unduly restrictive and will be omitted in the sequel. One may simply require of function $\mathbf{c}$ that the contraction properties of the auxiliary system do not depend on $\mathbf{d}(\mathbf{x}, t)$.
} 
switching systems [42]. Furthermore, two particular solutions are $\mathbf{y}=\mathbf{x}(t)$ and $\mathbf{y}=\mathbf{e}+y_{\infty} \mathbf{u}_{1}$, where $\mathbf{e}$ is a constant vector satisfying $(\mathbf{I}-\mathbf{W}) \mathbf{e}=\mathbf{b}$ and $y_{\infty}$ is a scalar variable defined by

$$
\tau \dot{y}_{\infty}+y_{\infty}=\lambda_{2} \mathbf{u}_{1}^{T}(\mathbf{x}-\mathbf{e})
$$

Thus, $\mathbf{x}(t)$ verifies exponentially the specific property that $(\mathbf{x}(t)-\mathbf{e})$ is aligned with $\mathbf{u}_{1}$. Hence all solutions of the original system converge exponentially to a line attractor of the form $\mathbf{x}=\mathbf{e}+x_{\infty} \mathbf{u}_{1}$, where $\dot{x}_{\infty}=0$ using the original $\mathbf{x}$ dynamics. The actual value of $x_{\infty}$ is determined by the initial conditions.

Note that contraction may be trivially regarded as a particular case of partial contraction.

\section{Two Coupled Oscillators}

In this section, we investigate coupled networks composed only by two oscillators, before generalizing the results in the next section.

\subsection{One-Way Coupling Configuration}

Consider a pair of one-way (unidirectional) coupled identical oscillators

$$
\left\{\begin{array}{l}
\dot{\mathbf{x}}_{1}=\mathbf{f}\left(\mathbf{x}_{1}, t\right) \\
\dot{\mathbf{x}}_{2}=\mathbf{f}\left(\mathbf{x}_{2}, t\right)+\mathbf{u}\left(\mathbf{x}_{1}\right)-\mathbf{u}\left(\mathbf{x}_{2}\right)
\end{array}\right.
$$

where $\mathbf{x}_{1}, \mathbf{x}_{2} \in \mathbb{R}^{m}$ are the state vectors, $\mathbf{f}\left(\mathbf{x}_{i}, t\right)$ the dynamics of the uncoupled oscillators, and $\mathbf{u}\left(\mathbf{x}_{1}\right)-\mathbf{u}\left(\mathbf{x}_{2}\right)$ the coupling force.

Theorem 2 If the function $\mathbf{f}-\mathbf{u}$ is contracting in (3), two systems $\mathbf{x}_{1}$ and $\mathbf{x}_{2}$ will reach synchrony exponentially regardless of the initial conditions.

Proof: The second subsystem, with $\mathbf{u}\left(\mathbf{x}_{1}\right)$ as input, is contracting, and $\mathbf{x}_{2}(t)=\mathbf{x}_{1}(t)$ is a particular solution.

Example 3.1: Consider two coupled identical Van der Pol oscillators

$$
\left\{\begin{array}{l}
\ddot{x}_{1}+\alpha\left(x_{1}^{2}-1\right) \dot{x}_{1}+\omega^{2} x_{1}=0 \\
\ddot{x}_{2}+\alpha\left(x_{2}^{2}-1\right) \dot{x}_{2}+\omega^{2} x_{2}=\alpha \kappa\left(\dot{x}_{1}-\dot{x}_{2}\right)
\end{array}\right.
$$

where $\alpha, \omega$ and $\kappa$ are strictly positive constants (this assumption will apply to all our Van der Pol examples). Since the system

$$
\ddot{x}+\alpha\left(x^{2}+\kappa-1\right) \dot{x}+\omega^{2} x=u(t)
$$

is semi-contracting for $\kappa>1$ (see Appendix), $x_{2}$ will synchronize to $x_{1}$ asymptotically.

Note that a typical engineering application with a one-way coupling configuration is observer design, in which case $\mathbf{x}_{1}$ represents the measurement. The result of Theorem 2 can be easily extended to a network containing $n$ oscillators with a chain structure (or more generally, a tree structure)

$$
\left\{\begin{array}{l}
\dot{\mathbf{x}}_{1}=\mathbf{f}\left(\mathbf{x}_{1}, t\right) \\
\dot{\mathbf{x}}_{2}=\mathbf{f}\left(\mathbf{x}_{2}, t\right)+\mathbf{u}\left(\mathbf{x}_{1}\right)-\mathbf{u}\left(\mathbf{x}_{2}\right) \\
\cdots \\
\dot{\mathbf{x}}_{n}=\mathbf{f}\left(\mathbf{x}_{n}, t\right)+\mathbf{u}\left(\mathbf{x}_{n-1}\right)-\mathbf{u}\left(\mathbf{x}_{n}\right)
\end{array}\right.
$$

where the synchronization condition is the same as that for system (3). 


\subsection{Two-Way Coupling Configuration}

The meaning of synchronization may vary in different contexts. In this paper, we define synchronization of two (or more) oscillators $\mathbf{x}_{1}, \mathbf{x}_{2}$ as corresponding to a complete match, i.e., $\mathbf{x}_{1}=\mathbf{x}_{2}$. Similarly, we define anti-synchronization as $\mathbf{x}_{1}=-\mathbf{x}_{2}$. These two behaviors are called in-phase synchronization and anti-phase synchronization in many communities.

\subsubsection{Synchronization}

Theorem 3 Consider two coupled systems. If the dynamics equations verify

$$
\dot{\mathbf{x}}_{1}-\mathbf{h}\left(\mathbf{x}_{1}, t\right)=\dot{\mathbf{x}}_{2}-\mathbf{h}\left(\mathbf{x}_{2}, t\right)
$$

where the function $\mathbf{h}$ is contracting, then $\mathbf{x}_{1}$ and $\mathbf{x}_{2}$ will converge to each other exponentially, regardless of the initial conditions.

Proof: Given initial conditions $\mathbf{x}_{1}(0)$ and $\mathbf{x}_{2}(0)$, denote by $\mathbf{x}_{1}(t)$ and $\mathbf{x}_{2}(t)$ the solutions of the two coupled systems. Define

$$
\mathbf{g}\left(\mathbf{x}_{1}, \mathbf{x}_{2}, t\right)=\dot{\mathbf{x}}_{1}-\mathbf{h}\left(\mathbf{x}_{1}, t\right)=\dot{\mathbf{x}}_{2}-\mathbf{h}\left(\mathbf{x}_{2}, t\right)
$$

and construct the auxiliary system

$$
\dot{\mathbf{y}}=\mathbf{h}(\mathbf{y}, t)+\mathbf{g}\left(\mathbf{x}_{1}(t), \mathbf{x}_{2}(t), t\right)
$$

This system is contracting since the function $\mathbf{h}$ is contracting, and therefore all solutions of $\mathbf{y}$ converge together exponentially. Since $\mathbf{y}=\mathbf{x}_{1}(t)$ and $\mathbf{y}=\mathbf{x}_{2}(t)$ are two particular solutions, this implies that $\mathbf{x}_{1}(t)$ and $\mathbf{x}_{2}(t)$ converge together exponentially.

Remarks

- Theorem 3 can also be proved by constructing another auxiliary system

$$
\left\{\begin{array}{l}
\dot{\mathbf{y}}_{1}=\mathbf{h}\left(\mathbf{y}_{1}, t\right)+\mathbf{g}\left(\mathbf{x}_{1}, \mathbf{x}_{2}, t\right) \\
\dot{\mathbf{y}}_{2}=\mathbf{h}\left(\mathbf{y}_{2}, t\right)+\mathbf{g}\left(\mathbf{x}_{1}, \mathbf{x}_{2}, t\right)
\end{array}\right.
$$

which has a particular solution verifying the specific property $\mathbf{y}_{1}=\mathbf{y}_{2}$. Since this auxiliary system is composed of two independent subsystems driven by the same inputs, the proof can be simplified as above by using a auxiliary system of reduced dimension.

- Theorem 2 is a particular case of Theorem 3. So is, for instance, a system of two-way coupled identical oscillators of the form

$$
\left\{\begin{array}{l}
\dot{\mathbf{x}}_{1}=\mathbf{f}\left(\mathbf{x}_{1}, t\right)+\mathbf{u}\left(\mathbf{x}_{2}\right)-\mathbf{u}\left(\mathbf{x}_{1}\right) \\
\dot{\mathbf{x}}_{2}=\mathbf{f}\left(\mathbf{x}_{2}, t\right)+\mathbf{u}\left(\mathbf{x}_{1}\right)-\mathbf{u}\left(\mathbf{x}_{2}\right)
\end{array}\right.
$$

In such a system $\mathbf{x}_{1}$ tends to $\mathbf{x}_{2}$ exponentially if $\mathbf{f}-2 \mathbf{u}$ is contracting. Furthermore, because the coupling forces vanish exponentially, both oscillators tend to their original limit cycle behavior, but with a common phase.

- Although contraction properties are central to the analysis, the overall system itself in general is not contracting, and the common phase of the steady states is determined by the initial conditions $\mathbf{x}_{1}(0)$ and $\mathbf{x}_{2}(0)$. This stresses the difference between contraction and partial contraction. 
- Theorem 3 can be easily extended to coupled discrete-time systems, using discrete versions [40] of contraction analysis, to coupled hybrid systems, and to coupled systems expressed by partial-differential-equations.

Example 3.2: Consider again two coupled identical Van der Pol oscillators

$$
\left\{\begin{array}{l}
\ddot{x}_{1}+\alpha\left(x_{1}^{2}-1\right) \dot{x}_{1}+\omega^{2} x_{1}=\alpha \kappa_{1}\left(\dot{x}_{2}-\dot{x}_{1}\right) \\
\ddot{x}_{2}+\alpha\left(x_{2}^{2}-1\right) \dot{x}_{2}+\omega^{2} x_{2}=\alpha \kappa_{2}\left(\dot{x}_{1}-\dot{x}_{2}\right)
\end{array}\right.
$$

One has

$$
\ddot{x}_{1}+\alpha\left(x_{1}^{2}+\kappa_{1}+\kappa_{2}-1\right) \dot{x}_{1}+\omega^{2} x_{1}=\ddot{x}_{2}+\alpha\left(x_{2}^{2}+\kappa_{1}+\kappa_{2}-1\right) \dot{x}_{2}+\omega^{2} x_{2}
$$

From Theorem 3 and the result in the Appendix, we know that these two oscillators will reach synchrony asymptotically if $\kappa_{1}+\kappa_{2}>1$ for non-zero initial conditions.

\subsubsection{Anti-Synchronization}

In a seminal paper [69] inspired by Turing's work [48, 80], Smale describes a mathematical model where two identical biological cells, inert by themselves, can be excited into oscillations through diffusion interaction across their membranes. Using Theorem 3, we can build a coupled system

$$
\left\{\begin{array}{l}
\dot{\mathbf{x}}_{1}=\mathbf{h}\left(\mathbf{x}_{1}, t\right)+\mathbf{u}\left(\mathbf{x}_{2}, t\right)-\mathbf{u}\left(\mathbf{x}_{1}, t\right) \\
\dot{\mathbf{x}}_{2}=\mathbf{h}\left(\mathbf{x}_{2}, t\right)+\mathbf{u}\left(\mathbf{x}_{1}, t\right)-\mathbf{u}\left(\mathbf{x}_{2}, t\right)
\end{array}\right.
$$

to describe Smale's model.

Theorem 4 If the uncoupled dynamics $\mathbf{h}$ in (6) is contracting and odd in $\mathbf{x}, \mathbf{x}_{1}+\mathbf{x}_{2}$ will converge to zero exponentially regardless of the initial conditions. Moreover, for non-zero initial conditions, $\mathbf{x}_{1}$ and $\mathbf{x}_{2}$ will oscillate and reach anti-synchrony if the system

$$
\dot{\mathbf{z}}=\mathbf{h}(\mathbf{z}, t)-2 \mathbf{u}(\mathbf{z}, t)
$$

has a stable limit-cycle.

Proof: Replace $\mathbf{x}_{2}$ by $-\mathbf{x}_{2}$ in Theorem 3 .

Example 3.3: Consider specifically Smale's example [69], where

$$
\mathbf{h}(\mathbf{x}, t)=\mathbf{A} \mathbf{x}+\left[\begin{array}{c}
-x_{1}^{3} \\
0 \\
0 \\
0
\end{array}\right] \quad \mathbf{u}(\mathbf{x})=\frac{1}{2} \mathbf{K}\left[\begin{array}{c}
x_{1} \\
x_{2} \\
x_{3} \\
x_{4}
\end{array}\right]
$$

with

$$
\mathbf{A}=\left[\begin{array}{cccc}
1+a & 1 & \gamma a & 0 \\
-1 & a & 0 & \gamma a \\
-\gamma a & 0 & 2 a & 0 \\
0 & -\gamma a & 0 & 2 a
\end{array}\right] \quad \mathbf{K}=\left[\begin{array}{cccc}
a & 0 & \gamma a & 0 \\
0 & a & 0 & \gamma a \\
-\gamma a & 0 & -2 a & 0 \\
0 & -\gamma a & 0 & -2 a
\end{array}\right]
$$

For $a<-1, \mathbf{h}$ has a negative definite Jacobian and thus is contracting, and $\mathbf{h}-2 \mathbf{u}$ yields a stable limitcycle, so that the two originally stable cells are excited into oscillations for non-zero initial conditions. Requiring in addition that $\sqrt{2}<\gamma<3 / 2$ guarantees that all the eigenvalues of $\mathbf{K}$ are distinct, real and strictly positive, so that $\mathbf{K}$ can be transformed into a diagonal diffusion matrix through a linear change of coordinates. 


\subsubsection{Oscillator-Death}

Inverse to Smale's effect, in the phenomenon called oscillator-death (or amplitude-death) $[1,2,59]$ oscillators stop oscillating and stabilize at constant steady states once they are coupled. Oscillatordeath happens if the overall dynamics is contracting and autonomous, since this implies that the system tends exponentially to a unique equilibrium.

Example 3.4: Couple two Van der Pol oscillators with asymmetric forces

$$
\left\{\begin{array}{l}
\ddot{x}_{1}+\alpha\left(x_{1}^{2}-1\right) \dot{x}_{1}+\omega^{2} x_{1}=\alpha \kappa\left(\dot{x}_{2}-\dot{x}_{1}\right) \\
\ddot{x}_{2}+\alpha\left(x_{2}^{2}-1\right) \dot{x}_{2}+\omega^{2} x_{2}=\alpha \kappa\left(-\dot{x}_{1}-\dot{x}_{2}\right)
\end{array}\right.
$$

where $\kappa>1$. By introducing new variables $y_{1}$ and $y_{2}$ as in the Appendix, we get a generalized Jacobian

$$
\mathbf{F}=\left[\begin{array}{cccc}
-\alpha\left(x_{1}^{2}+\kappa-1\right) & \omega & \alpha \kappa & 0 \\
-\omega & 0 & 0 & 0 \\
-\alpha \kappa & 0 & -\alpha\left(x_{2}^{2}+\kappa-1\right) & \omega \\
0 & 0 & -\omega & 0
\end{array}\right] \leq \mathbf{0}
$$

whose symmetric part is simply that of two uncoupled damped Van der Pol oscillators. Thus both systems will tend to zero asymptotically.

\subsection{Van der Pol Oscillators with General Couplings}

As a conclusion of this section, we now consider two identical Van der Pol oscillators coupled in a very general way:

$$
\left\{\begin{array}{l}
\ddot{x}_{1}+\alpha\left(x_{1}^{2}-1\right) \dot{x}_{1}+\omega^{2} x_{1}=\alpha\left(\gamma \dot{x}_{2}-\kappa \dot{x}_{1}\right) \\
\ddot{x}_{2}+\alpha\left(x_{2}^{2}-1\right) \dot{x}_{2}+\omega^{2} x_{2}=\alpha\left(\gamma \dot{x}_{1}-\kappa \dot{x}_{2}\right)
\end{array}\right.
$$

where $\alpha$ is a positive constant. It can be proved as above that, as long as the condition

$$
|\gamma|>1-\kappa
$$

is satisfied, $x_{1}$ converges to $x_{2}$ asymptotically for all $\gamma \geq 0$ while $x_{1}$ converges to $-x_{2}$ asymptotically for all $\gamma \leq 0$. Note that if $\gamma=0$ we get two independent stable subsystems. Both $x_{1}$ and $x_{2}$ tend to the origin, which can be considered as a continuous connection between $\gamma>0$ and $\gamma<0$.

Next we need to study the stable behavior of the coupled systems in order to make sure that if they keep oscillating or tend to a stationary equilibrium. Assume first that $\gamma>0$, we have

$$
\ddot{x}_{i}+\alpha\left(x_{i}^{2}-1\right) \dot{x}_{i}+\omega^{2} x_{i} \rightarrow \alpha(\gamma-\kappa) \dot{x}_{i} \quad i=1,2
$$

which gives the stable dynamics of $x_{1}$ and $x_{2}$ as

$$
\ddot{x}_{i}+\alpha\left(x_{i}^{2}+\kappa-\gamma-1\right) \dot{x}_{i}+\omega^{2} x_{i}=0 .
$$

This dynamic equation has a stable limit cycle if $\gamma>\kappa-1$ or a stable equilibrium point at origin otherwise. A similar result can be derived for $\gamma<0$, where $x_{1}$ and $x_{2}$ reach anti-synchrony if $\gamma<1-\kappa$ or tend to zero otherwise.

Also note that:

- Setting $\kappa=1, x_{1}$ and $x_{2}$ will keep oscillating for all $\gamma \neq 0$. Oscillator-death as a transition state between synchronized and anti-synchronized solutions does not exist except when $\gamma=0$. 
- In general, a positive value of $\gamma$ represents a force to drive synchrony while a negative value to drive anti-synchrony. Hence it is easy to understand the behavior of system (7) where the coupling to the first oscillator tries to synchronize but the coupling to the second tries to anti-synchronize, with equal strength. A neutral result is thus obtained. In fact, if we look at a coupled system with non-symmetric couplings

$$
\left\{\begin{array}{l}
\ddot{x}_{1}+\alpha\left(x_{1}^{2}-1\right) \dot{x}_{1}+\omega^{2} x_{1}=\alpha\left(\gamma_{1} \dot{x}_{2}-\kappa_{1} \dot{x}_{1}\right) \\
\ddot{x}_{2}+\alpha\left(x_{2}^{2}-1\right) \dot{x}_{2}+\omega^{2} x_{2}=\alpha\left(\gamma_{2} \dot{x}_{1}-\kappa_{2} \dot{x}_{2}\right)
\end{array}\right.
$$

the condition for oscillator-death is

$$
\kappa_{1}>1, \kappa_{2}>1,\left(\kappa_{1}-1\right)\left(\kappa_{2}-1\right) \geq\left(\gamma_{1}+\gamma_{2}\right)^{2} / 4 .
$$

- If we add extra diffusion coupling associated to the states $x_{1}$ and $x_{2}$ to system (8)

$$
\left\{\begin{array}{l}
\ddot{x}_{1}+\alpha\left(x_{1}^{2}-1\right) \dot{x}_{1}+\omega^{2} x_{1}=\alpha\left(\gamma \dot{x}_{2}-\kappa \dot{x}_{1}\right)+\alpha\left(\bar{\gamma} x_{2}-\bar{\kappa} x_{1}\right) \\
\ddot{x}_{2}+\alpha\left(x_{2}^{2}-1\right) \dot{x}_{2}+\omega^{2} x_{2}=\alpha\left(\gamma \dot{x}_{1}-\kappa \dot{x}_{2}\right)+\alpha\left(\bar{\gamma} x_{1}-\bar{\kappa} x_{2}\right)
\end{array}\right.
$$

where $\kappa$ and $\bar{\kappa}$ are both positive, the main result preserves as long as $\gamma \bar{\gamma}>0$. If the condition $|\gamma|>1-\kappa$ is satisfied, $x_{1}$ converges to $x_{2}$ asymptotically for all $\gamma \geq 0$ while $x_{1}$ converges to $-x_{2}$ asymptotically for all $\gamma \leq 0$. The second coupling term does not change the qualitative results (but only the amplitude and frequency of the final behavior) as long as

$$
\omega^{2}+\alpha(\bar{\kappa}-|\bar{\gamma}|)>0 \text {. }
$$

These results can be regarded as a global generalization of the dynamics analysis of two identical Van der Pol oscillators in [57, 72].

\section{Nonlinear Networked Systems}

Most coupled oscillators in the natural world are organized in large networks, such as pacemaker cells in heart, neural networks in brain, fireflies with synchronized flashes, crickets with synchronized chirping, etc. [73, 77]. System (4) represents such an instance with a chain structure. There are many other possible structures, such as e.g. the three symmetric ones illustrated in Figure 1.

(a)

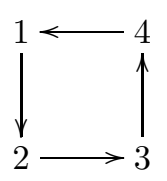

(b)

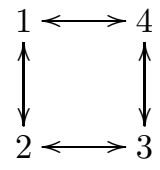

$(c)$

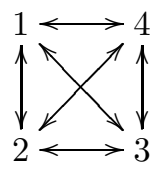

Figure 1: Networks with different symmetric structures $(n=4)$

In this section, we show that partial contraction analysis can be used to study synchronization in networks of nonlinear dynamic systems of various structures and arbitrary size. Coupling forces can be nonlinear as well. 


\subsection{Networks with All-To-All Symmetry}

Consider first a network with all-to-all symmetry, that is, with each element coupled to all the others. Such a network can be analyzed using an immediate extension of Theorem 3 .

Theorem $\mathbf{5}$ Consider $n$ coupled systems. If a contracting function $\mathbf{h}\left(\mathbf{x}_{i}, t\right)$ exists such that

$$
\dot{\mathbf{x}}_{1}-\mathbf{h}\left(\mathbf{x}_{1}, t\right)=\cdots=\dot{\mathbf{x}}_{n}-\mathbf{h}\left(\mathbf{x}_{n}, t\right)
$$

then all the systems will synchronize exponentially regardless of the initial conditions.

For instance, for identical oscillators coupled with diffusion-type force

$$
\dot{\mathbf{x}}_{i}=\mathbf{f}\left(\mathbf{x}_{i}, t\right)+\sum_{j=1}^{n}\left(\mathbf{u}\left(\mathbf{x}_{j}\right)-\mathbf{u}\left(\mathbf{x}_{i}\right)\right) \quad i=1,2, \ldots, n
$$

contraction of $\mathbf{f}-n \mathbf{u}$ guarantees synchronization of the whole network.

In [46], Mirollo and Strogatz study an all-to-all network of pulse-coupled integrate-and-fire oscillators, and derive a similar result on global synchronization.

\subsection{Networks with Less Symmetry}

Besides its direct application to all-to-all networks, Theorem 5 may also be used to study networks with less symmetry.

Example 4.1: Consider an $n=4$ network with two-way-ring symmetry (as illustrated in Figure 1(b))

$$
\dot{\mathbf{x}}_{i}=\mathbf{f}\left(\mathbf{x}_{i}, t\right)+\left(\mathbf{u}\left(\mathbf{x}_{i-1}\right)-\mathbf{u}\left(\mathbf{x}_{i}\right)\right)+\left(\mathbf{u}\left(\mathbf{x}_{i+1}\right)-\mathbf{u}\left(\mathbf{x}_{i}\right)\right) \quad i=1,2,3,4
$$

where the subscripts $i-1$ and $i+1$ are computed circularly. Combining these four oscillators into two groups $\left(\mathbf{x}_{1}, \mathbf{x}_{2}\right)$ and $\left(\mathbf{x}_{3}, \mathbf{x}_{4}\right)$, we find

$$
\left[\begin{array}{c}
\dot{\mathbf{x}}_{1}-\mathbf{f}\left(\mathbf{x}_{1}, t\right)+2 \mathbf{u}\left(\mathbf{x}_{1}\right) \\
\dot{\mathbf{x}}_{2}-\mathbf{f}\left(\mathbf{x}_{2}, t\right)+2 \mathbf{u}\left(\mathbf{x}_{2}\right)
\end{array}\right]=\left[\begin{array}{c}
\dot{\mathbf{x}}_{3}-\mathbf{f}\left(\mathbf{x}_{3}, t\right)+2 \mathbf{u}\left(\mathbf{x}_{3}\right) \\
\dot{\mathbf{x}}_{4}-\mathbf{f}\left(\mathbf{x}_{4}, t\right)+2 \mathbf{u}\left(\mathbf{x}_{4}\right)
\end{array}\right]=\left[\begin{array}{c}
\mathbf{u}\left(\mathbf{x}_{2}\right)+\mathbf{u}\left(\mathbf{x}_{4}\right) \\
\mathbf{u}\left(\mathbf{x}_{1}\right)+\mathbf{u}\left(\mathbf{x}_{3}\right)
\end{array}\right]
$$

Thus, if the function $\mathbf{f}-2 \mathbf{u}$ is contracting, $\left(\mathbf{x}_{1}, \mathbf{x}_{2}\right)$ converges to $\left(\mathbf{x}_{3}, \mathbf{x}_{4}\right)$ exponentially, and hence

$$
\left\{\begin{aligned}
\dot{\mathbf{x}}_{1}-\mathbf{f}\left(\mathbf{x}_{1}, t\right)+2 \mathbf{u}\left(\mathbf{x}_{1}\right) & \rightarrow 2 \mathbf{u}\left(\mathbf{x}_{2}\right) \\
\dot{\mathbf{x}}_{2}-\mathbf{f}\left(\mathbf{x}_{2}, t\right)+2 \mathbf{u}\left(\mathbf{x}_{2}\right) & \rightarrow 2 \mathbf{u}\left(\mathbf{x}_{1}\right)
\end{aligned}\right.
$$

so that in turn $\mathbf{x}_{1}$ converges to $\mathbf{x}_{2}$ exponentially if the function $\mathbf{f}-4 \mathbf{u}$ is contracting. The four oscillators then reach synchrony exponentially regardless of the initial conditions.

An extended partial contraction analysis can be used to study the example below, the idea of which will be generalized in the following section.

Definition 1 Consider $n$ square matrices $\mathbf{K}_{i}$ of identical dimensions, and a square symmetric matrix K. Define

$$
\mathbf{I}_{\mathbf{K}_{i}}^{n}=\left[\begin{array}{cccc}
\mathbf{K}_{1} & 0 & \cdots & 0 \\
0 & \mathbf{K}_{2} & \cdots & 0 \\
\vdots & \vdots & \ddots & \vdots \\
0 & 0 & \cdots & \mathbf{K}_{n}
\end{array}\right] \quad \mathbf{U}_{\mathbf{K}}^{n}=\left[\begin{array}{cccc}
\mathbf{K} & \mathbf{K} & \cdots & \mathbf{K} \\
\mathbf{K} & \mathbf{K} & \cdots & \mathbf{K} \\
\vdots & \vdots & \ddots & \vdots \\
\mathbf{K} & \mathbf{K} & \cdots & \mathbf{K}
\end{array}\right]_{n \times n}
$$

One has $\mathbf{I}_{\mathbf{K}_{i}}^{n}>\mathbf{0}$ if and only if $\mathbf{K}_{i}>\mathbf{0}, \forall i$, and $\mathbf{U}_{\mathbf{K}}^{n} \geq 0$ if and only if $\mathbf{K} \geq 0$. 
Example 4.2: Consider an $n=4$ network with one-way-ring symmetry (as illustrated in Figure 1(a))

$$
\dot{\mathbf{x}}_{i}=\mathbf{f}\left(\mathbf{x}_{i}, t\right)+\mathbf{K}\left(\mathbf{x}_{i-1}-\mathbf{x}_{i}\right) \quad i=1,2,3,4
$$

where $\mathbf{K}=\mathbf{K}^{T} \geq 0$ and the subscripts are calculated circularly. This system is equivalent to

$$
\dot{\mathbf{x}}_{i}=\mathbf{f}\left(\mathbf{x}_{i}, t\right)-\mathbf{K}\left(2 \mathbf{x}_{i}+\mathbf{x}_{i+1}+\mathbf{x}_{i+2}\right)+\mathbf{K} \sum_{j=1}^{4} \mathbf{x}_{j}
$$

Construct the auxiliary system

$$
\dot{\mathbf{y}}_{i}=\mathbf{f}\left(\mathbf{y}_{i}, t\right)-\mathbf{K}\left(2 \mathbf{y}_{i}+\mathbf{y}_{i+1}+\mathbf{y}_{i+2}\right)+\mathbf{K} \sum_{j=1}^{4} \mathbf{x}_{j}(t) \quad i=1,2,3,4
$$

The auxiliary system admits the particular solution $\mathbf{y}_{1}=\mathbf{y}_{2}=\mathbf{y}_{3}=\mathbf{y}_{4}=\mathbf{y}_{\infty}$, with

$$
\dot{\mathbf{y}}_{\infty}=\mathbf{f}\left(\mathbf{y}_{\infty}, t\right)-4 \mathbf{K} \mathbf{y}_{\infty}+\mathbf{K} \sum_{j=1}^{4} \mathbf{x}_{j}(t)
$$

To apply Theorem 1 for the specific property $\mathbf{y}_{1}=\mathbf{y}_{2}=\mathbf{y}_{3}=\mathbf{y}_{4}$ and prove that all $\mathbf{x}_{i}$ synchronize regardless of the initial conditions, there only remains to study the Jacobian matrix

$$
\mathbf{J}=\left[\begin{array}{cccc}
\mathbf{J}_{1}-2 \mathbf{K} & -\mathbf{K} & -\mathbf{K} & 0 \\
0 & \mathbf{J}_{2}-2 \mathbf{K} & -\mathbf{K} & -\mathbf{K} \\
-\mathbf{K} & 0 & \mathbf{J}_{3}-2 \mathbf{K} & -\mathbf{K} \\
-\mathbf{K} & -\mathbf{K} & 0 & \mathbf{J}_{4}-2 \mathbf{K}
\end{array}\right]
$$

where $\mathbf{J}_{i}=\frac{\partial \mathbf{f}}{\partial \mathbf{y}}\left(\mathbf{y}_{i}, t\right)$. The symmetric part of the Jacobian is

$$
\mathbf{J}_{s}=\mathbf{I}_{\left(\mathbf{J}_{i s}-\mathbf{K}\right)}^{4}-\frac{1}{2} \mathbf{U}_{\mathbf{K}}^{4}-\frac{1}{2} \mathbf{J}_{+} \quad \mathbf{J}_{+}=\left[\begin{array}{cccc}
\mathbf{K} & 0 & \mathbf{K} & 0 \\
0 & \mathbf{K} & 0 & \mathbf{K} \\
\mathbf{K} & 0 & \mathbf{K} & 0 \\
0 & \mathbf{K} & 0 & \mathbf{K}
\end{array}\right]
$$

We know that if $\forall i, \mathbf{J}_{i s}-\mathbf{K}<\mathbf{0}$, then $\mathbf{I}_{\left(\mathbf{J}_{i s}-\mathbf{K}\right)}^{4}<0$, and if $\mathbf{K} \geq 0$ then $\mathbf{U}_{\mathbf{K}}^{4} \geq 0$ and $\mathbf{J}_{+} \geq 0$. If both conditions are satisfied, the Jacobian $\mathbf{J}$ is negative definite and synchronization occurs.

Note that dynamics (10) is then contracting as well. This is not surprising since $\mathbf{y}_{1}=\mathbf{y}_{2}=\mathbf{y}_{3}=\mathbf{y}_{4}$ defines both an invariant set and a linear constraint, and linear constraints preserve contraction [40].

\subsection{Networks with General Structure}

Let us now move to networked systems under a very general coupling structure. For notational simplicity, we first assume that coupling forces are linear diffusive with gains $\mathbf{K}_{i j}$ (associated with coupling from node $i$ to $j$ ) positive definite, i.e., $\left(\mathbf{K}_{i j}\right)_{s}=\mathbf{K}_{i j s}>0$. We further assume that coupling links are bidirectional and symmetric in different directions, i.e., $\mathbf{K}_{i j}=\mathbf{K}_{j i}$. All these assumptions can be relaxed as we will show later.

Consider a network containing $n$ identical elements

$$
\dot{\mathbf{x}}_{i}=\mathbf{f}\left(\mathbf{x}_{i}, t\right)+\sum_{j \in \mathcal{N}_{i}} \mathbf{K}_{j i}\left(\mathbf{x}_{j}-\mathbf{x}_{i}\right) \quad i=1, \ldots, n
$$

where $\mathcal{N}_{i}$ denotes the set of indices of the active links of element $i$. It is equivalent to

$$
\dot{\mathbf{x}}_{i}=\mathbf{f}\left(\mathbf{x}_{i}, t\right)+\sum_{j \in \mathcal{N}_{i}} \mathbf{K}_{j i}\left(\mathbf{x}_{j}-\mathbf{x}_{i}\right)-\mathbf{K}_{0} \sum_{j=1}^{n} \mathbf{x}_{j}+\mathbf{K}_{0} \sum_{j=1}^{n} \mathbf{x}_{j}
$$


where $\mathbf{K}_{0}$ is chosen to be a constant symmetric positive definite matrix (we will discuss its function later). Again, construct an auxiliary system

$$
\dot{\mathbf{y}}_{i}=\mathbf{f}\left(\mathbf{y}_{i}, t\right)+\sum_{j \in \mathcal{N}_{i}} \mathbf{K}_{j i}\left(\mathbf{y}_{j}-\mathbf{y}_{i}\right)-\mathbf{K}_{0} \sum_{j=1}^{n} \mathbf{y}_{j}+\mathbf{K}_{0} \sum_{j=1}^{n} \mathbf{x}_{j}(t)
$$

which has a particular solution $\mathbf{y}_{1}=\cdots=\mathbf{y}_{n}=\mathbf{y}_{\infty}$ with

$$
\dot{\mathbf{y}}_{\infty}=\mathbf{f}\left(\mathbf{y}_{\infty}, t\right)-n \mathbf{K}_{0} \mathbf{y}_{\infty}+\mathbf{K}_{0} \sum_{j=1}^{n} \mathbf{x}_{j}(t)
$$

According to Theorem 1, if the auxiliary system (12) is contracting, then all system trajectories will verify the independent property $\mathbf{x}_{1}=\cdots=\mathbf{x}_{n}$ exponentially.

Next, we compute $\mathbf{J}_{s}$, the symmetric part of the Jacobian matrix of the auxiliary system.

Definition 2 Consider a square symmetric matrix $\mathbf{K}$, and define

$$
\mathbf{T}_{\mathbf{K}}^{n}=\left[\begin{array}{ccccc}
\ddots & \vdots & & \vdots & \\
\cdots & \mathbf{K} & \cdots & -\mathbf{K} & \cdots \\
& \vdots & \ddots & \vdots & \\
\cdots & -\mathbf{K} & \cdots & \mathbf{K} & \cdots \\
& \vdots & & \vdots & \ddots
\end{array}\right]_{n \times n}
$$

where all the elements in $\mathbf{T}_{\mathbf{K}}^{n}$ are zero except those displayed above at the four intersections of the $i$ th and $j$ th rows with the ith and $j$ th columns. One has $\mathbf{T}_{\mathbf{K}}^{n} \geq 0$ if $\mathbf{K} \geq 0$.

Letting $\mathcal{N}=\cup_{i=1}^{n} \mathcal{N}_{i}$ denote the set of active links in the network, define $\mathbf{L}_{\mathbf{K}}=\sum_{i, j \in \mathcal{N}} \mathbf{T}_{\mathbf{K}_{i j s}}^{n}$.

If we view the network as a graph, $\mathbf{L}_{\mathbf{K}}$ is the symmetric part of the weighted Laplacian matrix [18]. The standard laplacian matrix is denoted as $\mathbf{L}$. We can write

$$
\mathbf{J}_{s}=\mathbf{I}_{\mathbf{J}_{i s}}^{n}-\mathbf{L}_{\mathbf{K}}-\mathbf{U}_{\mathbf{K}_{0}}^{n}
$$

where $\mathbf{J}_{i s}=\left(\frac{\partial \mathbf{f}}{\partial \mathbf{y}}\left(\mathbf{y}_{i}, t\right)\right)_{s}$.

Lemma 1 Let

$$
\mathbf{J}_{r}=-\mathbf{L}_{\mathbf{K}}-\mathbf{U}_{\mathbf{K}_{0}}^{n}
$$

If $\mathbf{K}_{0}>\mathbf{0}, \mathbf{K}_{i j}>\mathbf{0}, \forall(i, j) \in \mathcal{N}$, and the network is connected, then $\mathbf{J}_{r}<\mathbf{0}$.

Proof: Note that each of the two parts in $\mathbf{J}_{r}$ is only negative semi-definite. Given an arbitrary nonzero vector $\mathbf{v}=\left[\mathbf{v}_{1}, \ldots, \mathbf{v}_{n}\right]^{T}$, one has

$$
\mathbf{v}^{T} \mathbf{J}_{r} \mathbf{v}=-\sum_{(i, j) \in \mathcal{N}}\left(\mathbf{v}_{i}-\mathbf{v}_{j}\right)^{T} \mathbf{K}_{i j s}\left(\mathbf{v}_{i}-\mathbf{v}_{j}\right)-\left(\sum_{i=1}^{n} \mathbf{v}_{i}\right)^{T} \mathbf{K}_{0}\left(\sum_{i=1}^{n} \mathbf{v}_{i}\right)<0
$$

because the condition that the network is connected guarantees that

$$
\mathbf{v}^{T} \mathbf{J}_{r} \mathbf{v}=0 \quad \text { if and only if } \quad \mathbf{v}_{1}=\cdots=\mathbf{v}_{n}=\mathbf{0} .
$$


Furthermore, the largest eigenvalue of $\mathbf{J}_{r}$ can be calculated as

$$
\lambda_{\max }\left(\mathbf{J}_{r}\right)=\max _{\|\mathbf{v}\|=1} \mathbf{v}^{T} \mathbf{J}_{r} \mathbf{v}=\max _{\|\mathbf{v}\|=1}\left(-\mathbf{v}^{T} \mathbf{L}_{\mathbf{K}} \mathbf{v}-\mathbf{v}^{T} \mathbf{U}_{\mathbf{K}_{0}}^{n} \mathbf{v}\right)
$$

Since $-\mathbf{v}^{T} \mathbf{U}_{\mathbf{K}_{0}}^{n} \mathbf{v}$ keeps decreasing as $\mathbf{K}_{0}$ increases except on the set $\sum_{i=1}^{n} \mathbf{v}_{i}=0$, we can choose $\mathbf{K}_{0}$ large enough and get

$$
\lambda_{\max }\left(\mathbf{J}_{r}\right)=-\min _{\substack{\|\mathbf{v}\|=1 \\ \sum_{i=1}^{n} \mathbf{v}_{i}=0}} \mathbf{v}^{T} \mathbf{L}_{\mathbf{K}} \mathbf{v}=-\lambda_{m+1}\left(\mathbf{L}_{\mathbf{K}}\right)
$$

according to the Courant-Fischer Theorem [25] - note that $\mathbf{K}_{0}$ is a virtual quantity used to make $\mathbf{J}_{r}<0$ in the partial contracting analysis, and thus it cannot affect the real system's synchronization rate. Here the eigenvalues are arranged in an increasing order, and $\lambda_{1}\left(\mathbf{L}_{\mathbf{K}}\right)=\cdots=\lambda_{m}\left(\mathbf{L}_{\mathbf{K}}\right)=0$, where $m$ is the dimension of each individual element.

Note that in the particular case that $m=1$ and all $\mathbf{K}_{i j}=1$, eigenvalue $\lambda_{2}\left(\mathbf{L}_{\mathbf{K}}\right)=\lambda_{2}(\mathbf{L})$ is a fundamental quantity in graph theory named algebraic connectivity [16], which is equal to zero if and only if the graph is not connected.

The above results imply immediately

Theorem 6 Regardless of initial conditions, all the elements within a generally coupled network (11) will reach synchrony or group agreement exponentially if

- the network is connected

- $\lambda_{\max }\left(\mathbf{J}_{\text {is }}\right)$ is upper bounded

- the couplings are strong enough

Specifically, the auxiliary system (12) is contracting if

$$
\lambda_{m+1}\left(\mathbf{L}_{\mathbf{K}}\right)>\max _{i} \lambda_{\max }\left(\mathbf{J}_{i s}\right) \quad \text { uniformly }
$$

Remarks

- The conditions given in Theorem 6 to guarantee synchronization represent requirements both on the individual systems' internal dynamics and the network's geometric structure. A lower bound on the corresponding threshold of the coupling strength can be computed through eigenvalue analysis for a specific network.

- Theorem 6 can also be used to find the threshold for symmetric subgroups in a network to reach synchrony, as in Example 4.1.

- Partial contraction analysis does not add any restriction on the uncoupled dynamics $\mathbf{f}(\mathbf{x}, t)$ other than requiring $\lambda_{\max }\left(\mathbf{J}_{i s}\right)$ to be upper bounded, which is easy to be satisfied if for instance individual elements are oscillators. As an example, $\lambda_{\max }\left(\mathbf{J}_{i s}\right)=\alpha$ for the Van der Pol oscillator. The dynamics $\mathbf{f}$ may have various qualitative properties, and it can be an oscillator, a contracting system, zero, or even a chaotic system [52, 67, 77]. For a group of contracting systems, if $\mathbf{\Theta}=\mathbf{I}$, the contraction property of the overall group will be enhanced by the diffusion couplings, and all the coupled systems are expected to converge to a common equilibrium point exponentially if $\mathbf{f}$ is autonomous. If $\boldsymbol{\Theta} \neq \mathbf{I}$, however, the situation is 
more complicated, and a transformation must be used before applying contraction analysis. The coupling gain may lose positivity through the transformation, and the stability of the equilibrium point may be destroyed with strong enough coupling strengths. This kind of bifurcation is interesting especially if the otherwise silent systems behave as oscillators after coupling, a phenomenon of Smale's cells [38, 69, 80]. A simple example when $n=2$ has been discussed in Section 3.2.2.

- The definition of the "neighbor" sets $\mathcal{N}_{i}$ is quite flexible. While it may be based simply on position proximity (neighbors within a certain distance of each node), it can be chosen to reflect many other factors. Gestalt psychology [61], for instance, suggests that in human visual perception, grouping occurs not only by proximity, but also by similarity, closure, continuity, common region and connectedness. The coupling strengths can also be specified flexibly. For instance, using Schoenberg/Micchelli's theorems on positive definite functions [45], they can be chosen as smooth functions based on sums of gaussians.

- Partial contraction theory is derived from contraction theory. Thus many results from $[40,66]$ apply directly. Consider for instance a coupled network with constraints

$$
\dot{\mathbf{x}}_{i}=\mathbf{f}\left(\mathbf{x}_{i}, t\right)+\mathbf{n}_{i}+\sum_{j \in \mathcal{N}_{i}} \mathbf{K}_{j i}\left(\mathbf{x}_{j}-\mathbf{x}_{i}\right) \quad i=1, \ldots, n
$$

where $\mathbf{n}_{i}$ represents a superimposed flow normal to the constraint manifold and has the same form to each system. Construct the corresponding auxiliary system

$$
\dot{\mathbf{y}}_{i}=\mathbf{f}\left(\mathbf{y}_{i}, t\right)+\mathbf{n}_{i}+\sum_{j \in \mathcal{N}_{i}} \mathbf{K}_{j i}\left(\mathbf{y}_{j}-\mathbf{y}_{i}\right)-\mathbf{K}_{0} \sum_{j=1}^{n} \mathbf{y}_{j}+\mathbf{K}_{0} \sum_{j=1}^{n} \mathbf{x}_{j}
$$

Using [40], contraction of the unconstrained flow (12) implies local contraction of the constrained flow (14), which means group agreement can be achieved for constrained network in a finite region which can be computed explicitly. In same cases, the introduction of the constraint combined with the specific property of the particular solution implies that the constrained original system is actually contracting. Similarly, because the auxiliary system is contracting, robustness results in [40] apply directly.

\subsection{Extensions}

Besides the properties discussed above, let us make a few more extensions to Theorem 6 , and relax assumptions made earlier.

\subsubsection{Nonlinear Couplings}

The analysis carries on straightforwardly to nonlinear couplings. For instance,

$$
\dot{\mathbf{x}}_{i}=\mathbf{f}\left(\mathbf{x}_{i}, t\right)+\sum_{j \in \mathcal{N}_{i}} \mathbf{u}_{j i}\left(\mathbf{x}_{j}, \mathbf{x}_{i}, \mathbf{x}, t\right)
$$

where the couplings are of the form

$$
\mathbf{u}_{j i}=\mathbf{u}_{j i}\left(\mathbf{x}_{j}-\mathbf{x}_{i}, \mathbf{x}, t\right)
$$

with $\mathbf{u}_{j i}(\mathbf{0}, \mathbf{x}, t)=\mathbf{0} \forall i, j, \mathbf{x}, t$. The whole proof is the same except that we define

$$
\mathbf{K}_{j i}=\frac{\partial \mathbf{u}_{j i}\left(\mathbf{x}_{j}-\mathbf{x}_{i}, \mathbf{x}, t\right)}{\partial\left(\mathbf{x}_{j}-\mathbf{x}_{i}\right)}>0 \quad \text { uniformly }
$$


and assume $\mathbf{K}_{j i}=\mathbf{K}_{i j}$.

For instance, one may have

$$
\mathbf{u}_{j i}=\left(\mathbf{C}_{j i}(t)+\mathbf{B}_{j i}(t)\left\|\mathbf{x}_{j}-\mathbf{x}_{i}\right\|\right)\left(\mathbf{x}_{j}-\mathbf{x}_{i}\right)
$$

with $\mathbf{C}_{j i}=\mathbf{C}_{i j}>0$ uniformly and $\mathbf{B}_{j i}=\mathbf{B}_{i j} \geq 0$, in which case we construct the auxiliary system as

$$
\dot{\mathbf{y}}_{i}=\mathbf{f}\left(\mathbf{y}_{i}, t\right)+\sum_{j \in \mathcal{N}_{i}}\left(\mathbf{C}_{j i}(t)+\mathbf{B}_{j i}(t)\left\|\mathbf{x}_{j}(t)-\mathbf{x}_{i}(t)\right\|\right)\left(\mathbf{y}_{j}-\mathbf{y}_{i}\right)-\mathbf{K}_{0} \sum_{j=1}^{n} \mathbf{y}_{j}+\mathbf{K}_{0} \sum_{j=1}^{n} \mathbf{x}_{j}(t)
$$

Note that if the network is all-to-all coupled, the coupling forces can be even more general as we discussed in Section 4.1.

\subsubsection{One-way Couplings}

The bidirectional coupling assumption on each link is not always necessary. Consider a coupled network with one-way-ring structure and linear diffusion coupling force

$$
\dot{\mathbf{x}}_{i}=\mathbf{f}\left(\mathbf{x}_{i}, t\right)+\mathbf{K}\left(\mathbf{x}_{i-1}-\mathbf{x}_{i}\right) \quad i=1, \ldots, n
$$

where by convention $i-1=n$ when $i=1$. We assume that the coupling gain $\mathbf{K}=\mathbf{K}^{T}>0$ is identical to all links. Hence,

$$
\mathbf{J}_{r}=-\frac{1}{2} \mathbf{L}_{\mathbf{K}}-\mathbf{U}_{\mathbf{K}_{0}}^{n}
$$

is negative definite with

$$
\mathbf{L}_{\mathbf{K}}=\sum_{i=1}^{n} \mathbf{T}_{\mathbf{K}}^{n}(i, i+1)
$$

Since

$$
\lambda_{m+1}\left(\frac{1}{2} \sum_{i=1}^{n} \mathbf{T}_{\mathbf{K}}^{n}(i, i+1)\right)=\frac{1}{2} \lambda_{\min }(\mathbf{K}) \lambda_{2}\left(\sum_{i=1}^{n} \mathbf{T}_{1}^{n}(i, i+1)\right)=\lambda_{\min }(\mathbf{K})\left(1-\cos \frac{2 \pi}{n}\right),
$$

the threshold to reach synchrony exponentially is

$$
\lambda_{\min }(\mathbf{K})\left(1-\cos \frac{2 \pi}{n}\right)>\max _{i} \lambda_{\max }\left(\mathbf{J}_{i s}\right) \quad \text { uniformly }
$$

A special case was given in Example 4.2 with $n=4$.

Thus, Theorem 6 can be extended to networks whose links are either bidirectional with $\mathbf{K}_{j i}=\mathbf{K}_{i j}$ or unidirectional but formed as rings with $\mathbf{K}^{T}=\mathbf{K}$ (where $\mathbf{K}$ is identical within the same ring but may differ between different rings). Synchronized groups with increasing complexity can be generated through accumulation of smaller groups.

Throughout the remainder of the paper, all results on bidirectionally coupled networks will apply to unidirectional rings as well.

\subsubsection{Positive Semi-Definite Couplings}

Theorem 6 requires definite coupling gains. If the $\mathbf{K}_{i j}$ are only positive semi-definite, additional conditions must be added to the uncoupled system dynamics to guarantee globally stable synchronization. 
Without loss of generality, we assume

$$
\mathbf{K}_{i j s}=\left[\begin{array}{cc}
\overline{\mathbf{K}}_{i j s} & 0 \\
0 & 0
\end{array}\right]
$$

where $\overline{\mathbf{K}}_{i j s}$ is positive definite and has a common dimension for all links. Thus, we can divide the uncoupled dynamics $\mathbf{J}_{i s}$ into the form

$$
\mathbf{J}_{i s}=\left[\begin{array}{cc}
\mathbf{J}_{11 s} & \mathbf{J}_{12} \\
\mathbf{J}_{12}^{T} & \mathbf{J}_{22 s}
\end{array}\right]_{i}
$$

with each component having the same dimension as that of the corresponding one in $\mathbf{K}_{i j s}$. A sufficient condition to guarantee globally stable synchronization behavior in the region beyond a coupling strength threshold is that, $\forall i, \mathbf{J}_{22 s}$ is contracting and both $\lambda_{\max }\left(\mathbf{J}_{11 s}\right), \sigma_{\max }\left(\mathbf{J}_{12}\right)$ are bounded.

Indeed, given an arbitrary vector $\mathbf{v}=\left(\mathbf{v}_{1}, \ldots, \mathbf{v}_{n}\right)$, one has

$$
\begin{aligned}
\mathbf{v}^{T} \mathbf{J}_{s} \mathbf{v}=\sum_{i=1}^{n} \mathbf{v}_{i}^{T} \mathbf{J}_{i s} \mathbf{v}_{i}+\mathbf{v}^{T} \mathbf{J}_{r} \mathbf{v} & \leq \sum_{i=1}^{n} \mathbf{v}_{i}^{T}\left[\begin{array}{cc}
0 & \mathbf{J}_{12} \\
\mathbf{J}_{12}^{T} & \mathbf{J}_{22 s}
\end{array}\right]_{i} \mathbf{v}_{i}+\sum_{i=1}^{n} \mathbf{v}_{i}^{T}\left[\begin{array}{cc}
\lambda \mathbf{I} & 0 \\
0 & 0
\end{array}\right] \mathbf{v}_{i} \\
& =\sum_{i=1}^{n} \mathbf{v}_{i}^{T}\left[\begin{array}{cc}
\lambda \mathbf{I} & \mathbf{J}_{12} \\
\mathbf{J}_{12}^{T} & \mathbf{J}_{22 s}
\end{array}\right]_{i} \mathbf{v}_{i}
\end{aligned}
$$

where

$$
\lambda=\lambda_{\max }\left(\overline{\mathbf{J}}_{r}\right)+\max _{i} \lambda_{\max }\left(\mathbf{J}_{11 s}\right)
$$

and $\overline{\mathbf{J}}_{r}$ is a new matrix by ruling out the rows and columns containing only zero in $\mathbf{J}_{r}$ (we set $\mathbf{K}_{0}$ to be positive semi-definite and have the same form as $\mathbf{K}_{i j s}$ ) and hence is negative definite. From feedback combination condition (2), we know that a negative $\lambda$ with absolute value large enough, a contracting $\mathbf{J}_{22 s}$ and a bounded $\sigma\left(\mathbf{J}_{12}\right)$ for all $i$ guarantee the contracting of $\mathbf{J}_{s}$. In fact, global contraction of $\mathbf{J}_{22 s}$ is a very important necessary condition, without which the synchronization can not occur in an unbounded parameter region. Pecora first pointed this out in $[3,53,54]$ using a new concept called desynchronizing bifurcation. Recently, [56] independently studied a similar phenomenon.

Example 4.3 : Consider a network composed of identical Van der Pol oscillators in a general structure. The dynamics of the $i$ th oscillator is given as

$$
\ddot{x}_{i}+\alpha\left(x_{i}^{2}-1\right) \dot{x}_{i}+\omega^{2} x_{i}=\sum_{j \in \mathcal{N}_{i}} \alpha \kappa\left(\dot{x}_{j}-\dot{x}_{i}\right)
$$

Using partial contracting analysis, we have

$$
\mathbf{J}_{s}=\mathbf{I}_{\mathbf{J}_{i s}}^{n}+\mathbf{J}_{r}=\mathbf{I}_{\mathbf{J}_{i s}}^{n}-\mathbf{L}_{\mathbf{K}}-\mathbf{U}_{\mathbf{K}_{0}}^{n}
$$

with

$$
\mathbf{J}_{i s}=\left[\begin{array}{cc}
\alpha\left(1-x_{i}^{2}\right) & 0 \\
0 & 0
\end{array}\right] \quad \mathbf{K}_{i j}=\mathbf{K}_{i j s}=\left[\begin{array}{cc}
\alpha \kappa & 0 \\
0 & 0
\end{array}\right]
$$

By ruling out the even rows and even columns in $\mathbf{J}_{s}$ where the components are all zero, we get a new result

with

$$
\overline{\mathbf{J}}_{s}=\mathbf{I}_{\overline{\mathbf{J}}_{i s}}^{n}-\mathbf{L}_{\overline{\mathbf{K}}}-\mathbf{U}_{\overline{\mathbf{K}}_{0}}^{n}
$$

$$
\overline{\mathbf{J}}_{i s}=\alpha\left(1-x_{i}^{2}\right), \quad \overline{\mathbf{K}}_{i j}=\overline{\mathbf{K}}_{i j s}=\alpha \kappa .
$$

The condition for $\overline{\mathbf{J}}_{s}$ to be negative definite is

$$
\frac{1}{\kappa}<\lambda_{2}\left(\sum_{i, j \in \mathcal{N}} \mathbf{T}_{1}^{n}\right)=\lambda_{2}(\mathbf{L})
$$


which guarantees simultaneously that $\mathbf{J}_{s}$ is negative semi-definite. Using semi-contracting analysis, we know that synchrony will happen asymptotically.

An important application of coupled nonlinear oscillators is the modeling of central pattern generators $[10$, 11, 19, 20, 21]. Consider a two-way-ring neural network composed of four identical Van der Pol oscillators as given in Figure 1(b). Assume that the first oscillator is connected to the left front leg while the third to the right back one. The system dynamics is given as

$$
\ddot{x}_{i}+\alpha\left(x_{i}^{2}-1\right) \dot{x}_{i}+\omega^{2} x_{i}=\alpha \kappa\left(\gamma_{(i-1) i} \dot{x}_{i-1}-\dot{x}_{i}\right)+\alpha \kappa\left(\gamma_{(i+1) i} \dot{x}_{i+1}-\dot{x}_{i}\right)
$$

with $i=1,2,3,4$. Choosing different values of coupling coefficient $\gamma_{i j}$, this model is able to generate rhythmic signals to drive different quadrupedal gaits. We set $\gamma_{i j}=\gamma_{j i}=1$ if we want the oscillators $i$ and $j$ to synchronize while set $\gamma_{i j}=\gamma_{j i}=-1$ if we want them to anti-synchronize. Thus, following the description of animal gaits in [10], we are able to realize the pace, trot, bound and pronk, the quadrupedal gaits which are highly symmetric and robust with relative phase lags of zero or half a period. For instance, the pace gait(left/right pairing) is achieved by setting

$$
\gamma_{41}=\gamma_{14}=-1, \quad \gamma_{21}=\gamma_{12}=1, \quad \gamma_{32}=\gamma_{23}=-1, \quad \gamma_{43}=\gamma_{34}=1
$$

and coupling gain $\kappa>\frac{1}{2}$. The convergence from one gait to another is global. Once all the $\gamma_{i j}$ are set to be zero, we get the stand.

A similar model can be used to study the locomotion of other numbers of legs. For instance, consider a two-way-ring network composed of six oscillators. By setting $\kappa>1$ and all the $\gamma_{i j}$ to be -1 , we are able to generate the tripod gait, a common hexapodal gait in which the front and rear legs on one side, and the middle leg on the other, move together, followed by the remaining three legs half a period later [11].

Example 4.4: The FitzHugh-Nagumo(FN) model [17, 49] is a well-known spiking-neuron model. Consider a diffusion-coupled network with $n$ identical FitzHugh-Nagumo neurons

$$
\left\{\begin{array}{l}
\dot{v}_{i}=c\left(v_{i}+w_{i}-\frac{1}{3} v_{i}^{3}+I\right)+\sum_{j \in \mathcal{N}_{i}} k_{j i}\left(v_{j}-v_{i}\right) \\
\dot{w}_{i}=-\frac{1}{c}\left(v_{i}-a+b w_{i}\right) \\
i=1, \ldots, n
\end{array}\right.
$$

where $a, b, c$ are strictly positive constants. Defining a transformation matrix $\Theta=\left[\begin{array}{ll}1 & 0 \\ 0 & c\end{array}\right]$, which leaves the coupling gain unchanged, yields the generalized Jacobian of the uncoupled dynamics

$$
\mathbf{F}_{i}=\left[\begin{array}{cc}
c\left(1-v_{i}^{2}\right) & 1 \\
-1 & -\frac{b}{c}
\end{array}\right]
$$

Thus the whole network will synchronize exponentially if

$$
\lambda_{2}\left(\sum_{(i, j) \in \mathcal{N}} \mathbf{T}_{k_{i j}}^{n}\right)=\lambda_{2}\left(\mathbf{L}_{\overline{\mathbf{K}}}\right)>c
$$

Note that the model can be generalized using a linear state transformation to a dimensionless system [48], with partial contraction analysis yielding a similar result.

\subsection{Algebraic Connectivity}

For a coupled network with given structure, increasing the coupling gain for a link or adding an extra link will both improve the synchronization process. In fact, these two operations are the same in a general understanding by adding an extra term $-\mathbf{T}_{\mathbf{K}_{i j s}}^{n}$ to the matrix $\mathbf{J}_{s}$. According to Weyl's Theorem [25], if square matrix $\mathbf{A}, \mathbf{B}$ are Hermitian and the eigenvalues $\lambda_{i}(\mathbf{A}), \lambda_{i}(\mathbf{B})$ and $\lambda_{i}(\mathbf{A}+\mathbf{B})$ are arranged in increasing order, for each $k=1,2, \ldots, n$, we have

$$
\lambda_{k}(\mathbf{A})+\lambda_{1}(\mathbf{B}) \leq \lambda_{k}(\mathbf{A}+\mathbf{B}) \leq \lambda_{k}(\mathbf{A})+\lambda_{n}(\mathbf{B})
$$


which means immediately

$$
\lambda_{k}\left(\mathbf{J}_{s}-\mathbf{T}_{\mathbf{K}_{i j s}}^{n}\right) \leq \lambda_{k}\left(\mathbf{J}_{s}\right)
$$

since $\lambda_{\max }\left(-\mathbf{T}_{\mathbf{K}_{i j s}}^{n}\right)=0$.

In fact, connecting each node to more neighbors is an effective way for large-size networks to lower the synchronization threshold. To see this in more detail, let us assume that all the links within the network are bidirectional (the corresponding graph is called undirected graph) with identical coupling gain $\mathbf{K}=\mathbf{K}^{T}>0$. Thus, according to [26] $\lambda_{m+1}\left(\mathbf{L}_{\mathbf{K}}\right)=\lambda_{2} \lambda_{\min }(\mathbf{K})$ where $\lambda_{2}$ is the algebraic connectivity of the standard Laplacian matrix. Denote

$$
\bar{\lambda}=\frac{\max _{i} \lambda_{\max }\left(\mathbf{J}_{i s}\right)}{\lambda_{\min }(\mathbf{K})}
$$

If both the individual element's uncoupled dynamics and the coupling gains are fixed, the synchronization condition (13) can be written as

$$
\lambda_{2}>\bar{\lambda} \quad \text { uniformly }
$$

We can further transform this condition to the ones based on more explicit properties in geometry. Given a graph $\mathbf{G}$ of order $n$, there exist lower bounds on its diameter ${ }^{2} d(\mathbf{G})$ and its mean distance ${ }^{3}$ $\bar{\rho}(\mathbf{G})[47]$

$$
\begin{aligned}
d(\mathbf{G}) & \geq \frac{4}{n \lambda_{2}} \\
(n-1) \bar{\rho}(\mathbf{G}) & \geq \frac{2}{\lambda_{2}}+\frac{n-2}{2}
\end{aligned}
$$

(these bounds are most informative when $\lambda_{2}$ is small) which in turn gives us lower bounds on algebraic connectivity

$$
\begin{aligned}
& \lambda_{2} \geq \frac{4}{n \cdot d(\mathbf{G})} \\
& \lambda_{2} \geq \frac{2}{(n-1) \bar{\rho}(\mathbf{G})-\frac{n-2}{2}}
\end{aligned}
$$

Thus, a sufficient condition to guarantee exponential network synchronization is

$$
d(\mathbf{G})<\frac{4}{n \bar{\lambda}}
$$

or

$$
\bar{\rho}(\mathbf{G})<\frac{2}{\bar{\lambda}(n-1)}+\frac{n-2}{2(n-1)}
$$

These results quantify the fact that different coupling links or nodes can make different contributions to synchronization, because they play different roles in network structure. In this sense, links between far-separated nodes contribute more than those between close neighbors, a fact also central to Small World models [83].

Example 4.5: In [29], Kopell and Ermentrout show that closed rings of oscillators will reliably synchronize with nearest-neighbor coupling, while open chains require nearest and next-nearest neighbor coupling. This result can be explained by assuming all gains are identical and expressing the synchronization condition (13) as

$$
\lambda_{\min }(\mathbf{K})>\frac{\max _{i} \lambda_{\max }\left(\mathbf{J}_{i s}\right)}{\lambda_{2}} \quad \text { uniformly }
$$

\footnotetext{
${ }^{2}$ Maximum number of links between two distinct vertices [18]

${ }^{3}$ Average number of links between distinct vertices [47]
} 
Assuming $n$ extremely large, for a graph with two-way-chain structure

$$
\lambda_{2}=2\left(1-\cos \left(\frac{\pi}{n}\right)\right) \approx 2\left(\frac{\pi}{n}\right)^{2}
$$

while for a graph with two-way-ring structure

$$
\lambda_{2}=2\left(1-\cos \left(\frac{2 \pi}{n}\right)\right) \approx 8\left(\frac{\pi}{n}\right)^{2}
$$

As illustrated in Figure 2, although the number of links only differ by one in these two cases, the effort to synchronize an open chain network is four times of that to a closed one.
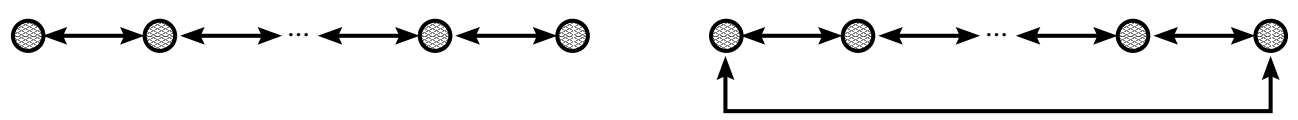

Figure 2: Comparison of a chain network and a ring.

Example 4.6: Consider a ring network, a star network and an all-to-all network (Figure 3) as network size $n$ tends to $\infty$. For the ring network, the coupling strength threshold for synchronization tends to infinity. For the star network it only needs to be of order 1, and for the all-to-all network it actually tends to 0 .

Thus, predictably, it is much easier to synchronize the star network than the ring. This is because the central node in the star network performs a global role, which keeps the graph diameter constant no matter how big the network size is. Such a star-liked structure is common. The internet, for instance, is composed of many connected subnetworks with star structures.
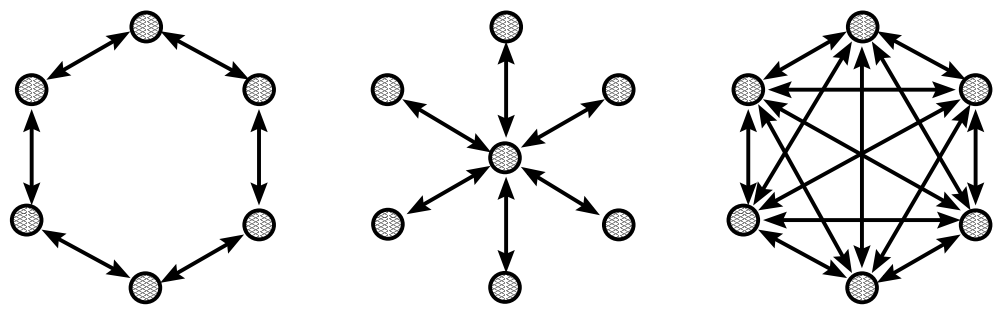

Figure 3: Comparison of three different kinds of networks.

This result is closely related to the Small World problem. Strogatz and Watts [76, 83, 84] showed that the average distance between nodes decreases with the increasing of the probability of adding short paths to each node. They also conjectured that synchronizability will be enhanced if the node is endowed with dynamics, which Barahona and Pecora showed numerically in [3].

\subsection{Fast Inhibition}

The dynamics of a large network of synchronized elements can be completely transformed by the addition of a single inhibitory coupling link. Start for instance with the synchronized network (11) and add a single inhibitory link between two arbitrary elements $a$ and $b$

$$
\begin{aligned}
& \dot{\mathbf{x}}_{a}=\mathbf{f}\left(\mathbf{x}_{a}, t\right)+\sum_{j \in \mathcal{N}_{a}} \mathbf{K}_{j a}\left(\mathbf{x}_{j}-\mathbf{x}_{a}\right)+\mathbf{K}\left(-\mathbf{x}_{b}-\mathbf{x}_{a}\right) \\
& \dot{\mathbf{x}}_{b}=\mathbf{f}\left(\mathbf{x}_{b}, t\right)+\sum_{j \in \mathcal{N}_{b}} \mathbf{K}_{j b}\left(\mathbf{x}_{j}-\mathbf{x}_{b}\right)+\mathbf{K}\left(-\mathbf{x}_{a}-\mathbf{x}_{b}\right)
\end{aligned}
$$


The symmetric part of the Jacobian matrix is

$$
\mathbf{J}_{s}=\mathbf{I}_{\mathbf{J}_{i s}}^{n}-\mathbf{L}_{\mathbf{K}}-\overline{\mathbf{T}}_{\mathbf{K}}^{n}
$$

where $\overline{\mathbf{T}}_{\mathbf{K}}^{n}$ is composed of zeroes except for four identical blocks

$$
\overline{\mathbf{T}}_{\mathbf{K}}^{n}=\left[\begin{array}{ccccc}
\ddots & \vdots & & \vdots & \\
\cdots & \mathbf{K} & \cdots & \mathbf{K} & \cdots \\
& \vdots & \ddots & \vdots & \\
\cdots & \mathbf{K} & \cdots & \mathbf{K} & \cdots \\
& \vdots & & \vdots & \ddots
\end{array}\right]_{n \times n}
$$

The matrix $\mathbf{J}_{r}^{*}=-\mathbf{L}_{\mathbf{K}}-\overline{\mathbf{T}}_{\mathbf{K}}^{n}$ is negative definite, since $\forall \mathbf{v} \neq 0$

$$
\mathbf{v}^{T} \mathbf{J}_{r}^{*} \mathbf{v}=-\sum_{(i, j) \in \mathcal{N}}\left(\mathbf{v}_{i}-\mathbf{v}_{j}\right)^{T} \mathbf{K}_{i j s}\left(\mathbf{v}_{i}-\mathbf{v}_{j}\right)-\left(\mathbf{v}_{a}+\mathbf{v}_{b}\right)^{T} \mathbf{K}\left(\mathbf{v}_{a}+\mathbf{v}_{b}\right)<0
$$

Thus, the network is contracting for strong enough coupling strengths. Hence, the $n$ elements will be inhibited. If the function $\mathbf{f}$ is autonomous, they will tend to equilibrium points. If the coupling strengths are not very strong, the inhibitory link will still have the ability to destroy the synchrony, and may then generate a desynchronized spiking sequence. Adding more inhibitory couplings preserves the result.

Such inhibition properties may be useful in pattern recognition to achieve rapid desynchronization between different objects. They may also be used as simplified models of minimal mechanisms for turning off unwanted synchronization, as e.g. in epileptic seizures or oscillations in internet traffic. In such applications, small and localized inhibition may also allow one to destroy unwanted synchronization while only introducing a small disturbance to the nominal behavior of the system. Cascades of inhibition are common in the brain, in a way perhaps reminiscent of NAND-based logic.

Note that the same effect can be achieved if we add self-inhibition to one (or more) arbitrary element. For instance,

$$
\dot{\mathbf{x}}_{a}=\mathbf{f}\left(\mathbf{x}_{a}, t\right)+\sum_{j \in \mathcal{N}_{a}} \mathbf{K}_{j a}\left(\mathbf{x}_{j}-\mathbf{x}_{a}\right)-\mathbf{K} \mathbf{x}_{a}
$$

In this case $\overline{\mathbf{T}}_{\mathbf{K}}^{n}$ is composed of zeroes except for one diagonal block

$$
\overline{\mathbf{T}}_{\mathbf{K}}^{n}=\left[\begin{array}{ccc}
\ddots & & \mathbf{0} \\
& \mathbf{K} & \\
\mathbf{0} & & \ddots
\end{array}\right]_{n \times n}
$$

\subsection{Switching Networks}

Closely related to oscillator synchronization, topics of collective behavior and group cooperation have also been the object of extensive recent research [7, 27, 34, 36, 37, 51, 60, 62, 68, 78, 79, 81]. A fundamental understanding of aggregate motions in the natural world, such as bird flocks, fish schools, animal herds, or bee swarms, for instance, would greatly help in achieving desired collective behaviors of artificial multi-agent systems, such as vehicles with distributed cooperative control rules. Since such networks are composed of moving units and each moving unit can only couple to its current neighbors, the network structure may change abruptly and asynchronously. 
Consider such a network

$$
\dot{\mathbf{x}}_{i}=\mathbf{f}\left(\mathbf{x}_{i}, t\right)+\sum_{j \in \mathcal{N}_{i}(t)} \mathbf{K}_{j i}\left(\mathbf{x}_{j}-\mathbf{x}_{i}\right) \quad i=1, \ldots, n
$$

where $\mathcal{N}_{i}(t)$ denotes the set of the active links associated with element $i$ at time $t$. Apply partial contraction analysis to each time interval during which the network structure $\mathcal{N}(t)$ is fixed. If

$$
\lambda_{m+1}\left(\mathbf{L}_{\mathbf{K}}\right)>\max _{i} \lambda_{\max }\left(\mathbf{J}_{i s}\right) \quad \text { uniformly } \forall \mathcal{N}(t),
$$

the auxiliary system (12) is always contracting, since $\delta \mathbf{y}^{T} \delta \mathbf{y}$ with $\delta \mathbf{y}=\left[\delta \mathbf{y}_{1}, \ldots, \delta \mathbf{y}_{n}\right]^{T}$ is continuous in time and upper bounded by a vanishing exponential (though its time-derivative can be discontinuous at discrete instants). Since the particular solution of the auxiliary system in each time interval is $\mathbf{y}_{1}=\cdots=\mathbf{y}_{n}=\mathbf{y}_{\infty}$, these $n$ elements will reach synchrony exponentially as they tend to $\mathbf{y}_{1}=\cdots=\mathbf{y}_{n}$ which is a constant region in the state-space. The threshold phenomenon described by inequality (17) is also reminiscent of phase transitions in physics [55] and of Bose-Einstein condensation [28] .

Example 4.7: Consider a simplified model of schooling or flocking in continuous-time with $\mathbf{f}=0$

$$
\dot{\mathbf{x}}_{i}=\sum_{j \in \mathcal{N}_{i}(t)} \mathbf{K}_{j i}\left(\mathbf{x}_{j}-\mathbf{x}_{i}\right) \quad i=1, \ldots, n
$$

where $\mathbf{x}_{i} \in \mathbb{R}^{m}$ denotes the states needed to reach agreements such as a vehicle's heading, attitude, velocity, etc. $\mathcal{N}_{i}(t)$ is defined for instance as the set of the nearest neighbors within a certain distance around element $i$ at current time $t$. The coupling gain $\mathbf{K}_{j i}$ satisfies those assumptions proposed in Section 4.3 and 4.4, i.e., the links are either bidirectional or unidirectional formed in rings. Since $\mathbf{J}_{i s}=0$ here, condition (17) is satisfied if only the network is connected. Therefore $\forall i, \mathbf{x}_{i}$ converges exponentially to a particular solution, which in this case is a constant value $\overline{\mathbf{x}}=\frac{1}{n} \sum_{i=1}^{n} \mathbf{x}_{i}(0)$. In fact, the network (18) need not be connected for any $t \geq 0$. A generalized condition can be derived [68] which is the same as that obtained in [27] for a discrete-time model.

Note that in the case of heading agreement based on spatial proximity, the issue of chattering is immaterial since switching cannot occur infinitely fast, while in the general case it can be avoided simply by using smooth transitions in time or space.

Finally note that, transposed to a neural network context, the overall convergence to $\overline{\mathbf{x}}$ can be interpreted as implementing a type of population coding.

\subsection{Leader-Followers Network}

In a network composed of peers, the phase of the collective behavior is hard to predict, since it depends on the initial conditions of all the coupled elements. To let the whole network converge to a specific trajectory, a "leader" can be added [27, 36].

Consider the dynamics of a coupled network

$$
\begin{aligned}
\dot{\mathbf{x}}_{0} & =\mathbf{f}\left(\mathbf{x}_{0}, t\right) \\
\dot{\mathbf{x}}_{i} & =\mathbf{f}\left(\mathbf{x}_{i}, t\right)+\sum_{j \in \mathcal{N}_{i}} \mathbf{K}_{j i}\left(\mathbf{x}_{j}-\mathbf{x}_{i}\right)+\gamma_{i} \mathbf{K}_{0 i}\left(\mathbf{x}_{0}-\mathbf{x}_{i}\right) \quad i=1, \ldots, n
\end{aligned}
$$

where $\mathbf{x}_{0}$ is the state of the leader, whose dynamics is independent, and $\mathbf{x}_{i}$ the state of the $i$ th follower. $\gamma_{i}$ is equal to either 0 or 1 and represents the connection from the leader to the followers. $\mathcal{N}_{i}$ denotes the set of peer-neighbors of element $i$, i.e., it does not include the possible link from $\mathbf{x}_{0}$ to $\mathbf{x}_{i}$. 
Theorem 7 Regardless of initial conditions, the states of all the followers within a generally coupled network (19) will converge exponentially to the state of the leader if

$$
\lambda_{\min }\left(\mathbf{L}_{\mathbf{K}}+\mathbf{I}_{\gamma_{i} \mathbf{K}_{0 i s}}^{n}\right)>\max _{i} \lambda_{\max }\left(\mathbf{J}_{i s}\right) \quad \text { uniformly }
$$

Proof: Since the dynamics of $\mathbf{x}_{0}$ is independent, we can treat it as an external input to the rest of the network, whose Jacobian matrix has the symmetric part

$$
\mathbf{J}_{s}=\mathbf{I}_{\mathbf{J}_{i s}}^{n}-\mathbf{L}_{\mathbf{K}}-\mathbf{I}_{\gamma_{i} \mathbf{K}_{0 i s}}^{n}
$$

The matrix $\mathbf{J}_{r}^{*}=-\mathbf{L}_{\mathbf{K}}-\mathbf{I}_{\gamma_{i} \mathbf{K}_{0 i s}}^{n}$ is negative definite if the augmented network with $n+1$ elements is connected. In fact, $\forall \mathbf{v} \neq 0$,

$$
\mathbf{v}^{T} \mathbf{J}_{r}^{*} \mathbf{v}=-\sum_{(i, j) \in \mathcal{N}}\left(\mathbf{v}_{i}-\mathbf{v}_{j}\right)^{T} \mathbf{K}_{i j s}\left(\mathbf{v}_{i}-\mathbf{v}_{j}\right)-\sum_{i=1}^{n} \gamma_{i}\left(\mathbf{v}_{i}^{T} \mathbf{K}_{0 i s} \mathbf{v}_{i}\right)<0
$$

Thus the system $\left[\mathbf{x}_{1}, \ldots, \mathbf{x}_{n}\right]^{T}$ is contracting if the coupling strengths are so strong that the condition (20) is true. Therefore, all solutions will converge to the particular one

$$
\mathbf{x}_{1}=\cdots=\mathbf{x}_{n}=\mathbf{x}_{0}
$$

exponentially regardless of the initial conditions. This result can be viewed as a generalization of Theorem 2.

\section{Remarks}

- For nonnegative $\max \lambda_{\max }\left(\mathbf{J}_{i s}\right)$, a necessary condition to realize leader-following is that the whole network of $n+1$ elements is connected. Thus the $n$ followers $\mathbf{x}_{1}, \ldots, \mathbf{x}_{n}$ could be either connected together, or there could be isolated subgroups all connected to the leader. Similar to the result in the previous section, the network structure of a leader-followers group does not have to be fixed during the whole time, neither.

- Comparing conditions (13) and (20) shows that, predictably, the existence of an additional leader does not always help the followers' network to reach agreement. But it does so if

$$
\lambda_{\min }\left(\mathbf{L}_{\mathbf{K}}+\mathbf{I}_{\gamma_{i} \mathbf{K}_{0 i s}}^{n}\right)>\lambda_{m+1}\left(\mathbf{L}_{\mathbf{K}}\right)
$$

Example 4.8: Consider for instance the case when the leader has identical connections to all other elements, $\forall i, \mathbf{K}_{0 i}=k \mathbf{I}, \quad k>0$. Then

$$
\lambda_{\min }\left(\mathbf{L}_{\mathbf{K}}+\mathbf{I}_{\gamma_{i}}^{n} \mathbf{K}_{0 i s}\right)=\min _{\|\mathbf{v}\|=1} \mathbf{v}^{T}\left(\mathbf{L}_{\mathbf{K}}+\mathbf{I}_{k \mathbf{I}}^{n}\right) \mathbf{v}=k
$$

This means the connections between the leader and the followers do promote the convergence within the followers' network if $\lambda_{m+1}\left(\mathbf{L}_{\mathbf{K}}\right)<k$, which is more likely to occur in a network with less connectivity.

- The connectivity of the followers' network helps the following process, which can be seen by applying Weyl's Theorem [25],

$$
\lambda_{i}\left(\mathbf{L}_{\mathbf{K}}+\mathbf{I}_{\gamma_{i} \mathbf{K}_{0 i s}}^{n}\right) \geq \lambda_{i}\left(\mathbf{I}_{\gamma_{i} \mathbf{K}_{0 i s}}^{n}\right) \quad i=1, \ldots, m n
$$


- The leader does not have to be single, but it can be a group of leading elements. In addition, in some cases it may receive feedback from the followers as well. This is the case in synchronization propagation, where the node density is unevenly distributed through the network. Since the synchronization rate depends on network connectivity, a high-density region will synchronize very quickly despite disturbances from other parts of the network. The inputs from these leaders then facilitate synchronization in low-density regions, where the elements may not be able to synchronize by themselves. A simple simulation was given in [68], and [88] observed a similar phenomenon by setting different interior connection weights inside different subgroups. Note that the leaders group here is very similar to the concept of core group in infectious disease dynamics [44], which is a group of the most active individuals. A small change in the core group will make a big difference in whether or not an epidemic can occur in the whole population.

- Synchronization can be made to propagate from the center outward in a more active way, for instance, through diffusion of a chemical produced by leaders or high-level elements and having the ability to expand the communication channels it passes through, i.e., to increase the gains through diffusion. Such a mechanism represents a hierarchical combination with gain dynamics. By extending the state, the analysis tools provided here can apply more generally to combinations where the gain dynamics are coupled to each other (with arbitrary connectivity) and to the $\mathbf{x}_{i}$.

- Different leaders $\mathbf{x}_{0}^{j}$ of arbitrary dynamics can define different primitives which can be combined. Contraction of the followers' dynamics $(i=1, \ldots, n)$

$$
\dot{\mathbf{x}}_{i}=\mathbf{f}\left(\mathbf{x}_{i}, t\right)+\sum_{j \in \mathcal{N}_{i}} \mathbf{K}_{j i}\left(\mathbf{x}_{j}-\mathbf{x}_{i}\right)+\sum_{j} \alpha_{j}(t) \gamma_{i}^{j} \mathbf{K}_{0 i}^{j}\left(\mathbf{x}_{0}^{j}-\mathbf{x}_{i}\right)
$$

is preserved if $\sum_{j} \alpha_{j}(t) \geq 1, \forall t \geq 0$.

- Besides its dubious moral implications, Theorem 7 also means that it is easy to detract a group from its nominal behavior by introducing a "covert" element, with possible applications to group control games, ethology, and animal and plant mimicry.

- Having a leader in a moving formation may yield other advantages, such as energy saving in aerodynamics [14, 62].

\section{Concluding Remarks}

We present a general method to analyze the dynamics of coupled nonlinear oscillators. Compared to previous studies (most of which were based on linearized models for coupled networks with limited size or particular structure), the results here are exact and global. The method can be used to study coupled networks with various structure and arbitrary size. An explicit synchronization condition is given for a generalized distributed network with diffusive couplings. The effect of a network's geometric property on its synchronization rate is discussed. Synchronizations condition for switching networks and for leader-followers networks are also provided. In fact, the results are not just limited to coupled oscillators, but apply to any coupled identical dynamic systems.

We introduce the concept of partial contraction, which investigates stability with respect to a specific behavior or property, and therefore can be very powerful to analyze large-scale systems. Based on contraction and/or partial contraction properties, synchronization analysis is greatly simplified by isolating the desired convergence behavior from the overall system dynamics. Furthermore, because it is virtual, the auxiliary, meta-system y can actually be centralized. Although this paper focuses mainly on identical properties of subsystem states, future applications of partial contraction 
to synchronization should investigate convergence to more general properties, such as phase locking in locomotion systems. The main limitation of the method is that the construction of the auxiliary system itself is not systematic.

Our current work includes the analysis of time-delayed communications in coupled networks, and of global convergence through local adaptation in networks of disparate dynamic elements.

Partial contraction analysis could also be applied in the context of discrete-time systems, hybrid systems or switching systems. It could allow one to study, for instance, the synchronization of pulse-coupled neurons in a distributed network, a widely-used model in computational neuroscience which still lacks a complete theoretical explanation.

Finally, the results presented in this paper could be extended to study systems described by nonlinear partial differential equations such as reaction-diffusion equations, and to the case when connections occur stochastically. The principle of a virtual centralized system may also have applications in quantum physics.

Acknowledgments: This work was supported in part by grants from the National Institutes of Health and the National Science Foundation. The authors would like to thank an anonymous reviewer for valuable comments and suggestions.

\section{Appendix: Driven damped Van der Pol oscillator}

Consider the second-order system

$$
\ddot{x}+\left(\beta+\alpha x^{2}\right) \dot{x}+\omega^{2} x=u(t)
$$

driven by an external input $u(t)$, where $\alpha, \beta, \omega$ are strictly positive constants. Introducing a new variable $y$, this system can be written

$$
\left\{\begin{array}{l}
\dot{x}=\omega y-\frac{\alpha}{3} x^{3}-\beta x \\
\dot{y}=-\omega x+\frac{u(t)}{\omega}
\end{array}\right.
$$

The corresponding Jacobian matrix

$$
\mathbf{F}=\left[\begin{array}{cc}
-\left(\beta+\alpha x^{2}\right) & \omega \\
-\omega & 0
\end{array}\right]
$$

is negative semi-definite [12]. Therefore,

$$
\frac{d}{d t}\left(\delta \mathbf{z}^{T} \delta \mathbf{z}\right)=2 \delta \mathbf{z}^{T} \mathbf{F} \delta \mathbf{z} \leq 0
$$

where $\delta \mathbf{z}=[\delta x, \delta y]^{T}$ is the generalized virtual displacement. Thus $\delta \mathbf{z}^{T} \delta \mathbf{z}$ tends to a lower limit asymptotically. Now check its higher-order Taylor expansion:

if $\delta x \neq 0$,

$$
\delta \mathbf{z}^{T} \delta \mathbf{z}(t+d t)-\delta \mathbf{z}^{T} \delta \mathbf{z}(t)=-2\left(\beta+\alpha x^{2}\right)(\delta x)^{2} d t+O\left((d t)^{2}\right)
$$

while if $\delta x=0$,

$$
\delta \mathbf{z}^{T} \delta \mathbf{z}(t+d t)-\delta \mathbf{z}^{T} \delta \mathbf{z}(t)=-4\left(\beta+\alpha x^{2}\right)(\delta \dot{x})^{2} \frac{(d t)^{3}}{3 !}+O\left((d t)^{4}\right)
$$

So the fact that $\delta \mathbf{z}^{T} \delta \mathbf{z}$ tends to a lower limit implies that $\delta x$ and $\delta \dot{x}$ both tend to 0 . System (21) is called semi-contracting, and for any external input all its solutions converge asymptotically to a single trajectory, independent of the initial conditions. Note that if $\beta<0$ and $u(t)=0$, system (21) has a unique, stable limit cycle. 


\section{References}

[1] Aronson, D.G., Ermentrout, G.B., and Kopell, N. (1990) Amplitude response of coupled oscillators, Physica D, 41:403-449

[2] Bar-Eli, K. (1985) On the Stability of Coupled Chemical Oscillators, Physica D, 14:242-252

[3] Barahona, M., and Pecora, L.M. (July 2002) Synchronization in Small-world Systems, Physical Review Letters

[4] Bay, John S., and Hemami, H. (April 1987) Modeling of a Neural Pattern Generator with Coupled Nonlinear Oscillators, IEEE Transactions on Biomedical Engineering

[5] Bressloff, P.C., Coombes, S., Souza, B. de (October 1997) Dynamics of a Ring of Pulse-Coupled Oscillators: Group Theoretic Approach, Physical Review Letters

[6] Brody, C.D., and Hopfield, J.J. (2003) Simple Networks for Spike-Timing-Based Computation, with Application to Olfactory Processing, Neuron, 37:843-852

[7] Bruckstein, A.M., Mallows, C.L., and Wagner, I.A. (1997) Cooperative Cleaners: a Study in Ant-Robotics, American Mathematical Monthly, 104(4): 323-343

[8] Chakraborty, T., Rand, R.H. (1988) The Transition from Phase Locking to Drift in a System of Two Weakly Coupled Van der Pol Oscillators, International J. Nonlinear Mechanics, 23:369-376

[9] Chua, L.O. (1998) CNN: a paradigm for complexity, Singapore; Hong Kong: World Scientific

[10] Collins, J.J., and Stewart, I.N. (1993) Coupled Nonlinear Oscillators and the Symmetries of Animal Gaits, Nonlinear Science, 3:349-392

[11] Collins, J.J., and Stewart, I.N. (1993) Hexapodal Gaits and Coupled Nonlinear Oscillator Models, Biological Cybernetics, 68:287-298

[12] Combescot, C., and Slotine, J.J.E. (2000) A Study of Coupled Oscillators, MIT Nonlinear Systems Laboratory, Report MIT-NSL-000801

[13] Coombes, S. (2001) Phase-locking in networks of pulse-coupled McKean relaxation oscillators, Physica D

[14] Cutts, C., and Speakman, J. (1994) Energy Savings in Formation flight of Pink-Footed Geese, J. Exp. Biol., 189:251-261

[15] Dragoi, V., and Grosu, I. (1998) Synchronization of Locally Coupled Neural Oscillators, Neural Processing Letters, 7

[16] Fiedler, M., (1973) Algebraic Connectivity of Graphs, Czechoslovak Mathematical Journal, 23(98):298-305

[17] FitzHugh, R.A. (1961) Impulses and Physiological States in Theoretical Models of Nerve Membrane, Biophys. $J ., 1: 445-466$

[18] Godsil, C., and Royle, G. (2001) Algebraic Graph Theory, Springer

[19] Golubitsky, M., Stewart, I., Buono, P.L., and Collins, J.J. (1998) A modular Network for Legged Locomotion, Physica D., 115:56-72

[20] Golubitsky, M., Stewart, I., Buono, P.L., and Collins, J.J. (1999) The Role of Symmetry in Locomotor Central Pattern Generators and Animal Gaits, Nature, 401

[21] Golubitsky, M., and Stewart, I. (2002) Patterns of Oscillation in Coupled Cell Systems, Geometry, Dynamics, and Mechanics: 60th Birthday Volume for J.E. Marsden, 243-286, Springer-Verlag.,

[22] Hahnloser, R.H., Seung, H.S., and Slotine. J.J.E. (2003) Permitted And Forbidden Sets in Symmetric ThresholdLinear Networks, Neural Computation 15:621-38

[23] Hirsch, M., and Smale, S. (1974) Differential Equations, Dynamical Systems, and Linear Algebra, Academic Press, New York

[24] Hopfield, J.J., and Brody, C.D. (2001) What Is A Moment? Transient Synchrony as a Collective Mechanism for Spatiotemporal Integration, Proc. Natl. Acad. Sci. USA, 98:1282-1287

[25] Horn, R.A., and Johnson, C.R. (1985) Matrix Analysis, Cambridge University Press

[26] Horn, R.A., and Johnson, C.R. (1989) Topics in Matrix Analysis, Cambridge University Press

[27] Jadbabaie, A., Lin, J., and Morse, A.S. (May 2003) Coordination of Groups of Mobile Autonomous Agents Using Nearest Neighbor Rules, IEEE Transactions on Automatic Control

[28] Ketterle, W. (2002) When atoms behave as waves: Bose-Eintein condensation and the atom laser, Rev. Mod. Phys., 74

[29] Kopell, N., and Ermentrout, G.B. (1986) Symmetry and Phase-locking in Chains of Weakly Coupled Oscillators, Communications on Pure and Applied Mathematics, 39:623-660

[30] Kopell, N., and Somers, D.C. (1995) Anti-phase Solutions in Relaxation Oscillators Coupled Through Excitatory Interactions, Journal of Mathematical Biology, 33

[31] Kopell, N. (2000) We got rhythm: Dynamical Systems of the Nervous System, published version of the 1998 Gibbs Lecture of the AMS, Notices of the AMS, 47:6-16

[32] Krishnaprasad, P.S., and Tsakiris, D. (2001) Oscillations, SE(2)-Snakes and Motion Control: A Study of the Roller Racer, Dyanmics and Stability of Systems

[33] Kuramoto, Y. (1984) Chemical Oscillations, Wave, and Turbulence, Springer, Berlin

[34] Langbort, C., and D'Andrea, R. (2003) Distributed Control of Spatially Reversible Interconnected Systems with 
Boundary Conditions, submitted to SIAM Journal on Control and Optimization

[35] Latham, P.E., Deneve, S., and Pouget, A. (2003) Optimal Computation With Attractor Networks, J. Physiol. (Paris), 97:683-694

[36] Leonard, N.E., and Fiorelli, E. (2001) Virtual Leaders, Artificial Potentials and Coordinated Control of Groups, 40th IEEE Conference on Decision and Control

[37] Lin, Z., Broucke, M., and Francis, B. (2003) Local Control Strategies for Groups of Mobile Autonomous Agents, submitted to IEEE Trans. Automatic Control

[38] Loewenstein, Y., and Sompolinsky, H. (2002) Oscillations by Symmetry Breaking in Homogeneous Networks with Electrical Coupling, Physical Review E, 65:1-11

[39] Lohmiller, W. (1999) Contraction Analysis of Nonlinear Systems, Ph.D. Thesis, Department of Mechanical Engineering, MIT

[40] Lohmiller, W., and Slotine, J.J.E. (1998) On Contraction Analysis for Nonlinear Systems, Automatica, 34(6)

[41] Lohmiller, W., and Slotine, J.J.E. (2000) Control System Design for Mechanical Systems Using Contraction Theory, IEEE Trans. Aut. Control, 45(5)

[42] Lohmiller, W., and Slotine, J.J.E. (2000) Nonlinear Process Control Using Contraction Theory A.I.Ch.E. Journal, March 2000.

[43] Manor, Y., Nadim, F., Ritt, J., Epstein, S., Marder, E., Kopell, N. (1999) Network Oscillations Generated by Balancing Asymmetric Inhibition in Passive Neurons, Journal of Neuroscience., 19

[44] May, R.M., Gupta, S., and McLean, A.R. (2001) Infectious Disease Dynamics: What Characterizes a Successful Invader? Phil. Trans. R. Soc. Lond. B, 356:901-910

[45] Micchelli, C.A. (1986) Interpolation of Scattered Data, Constructive Approximation, 2:11-22.

[46] Mirollo, R.E., Strogatz, S.H. (December 1990) Synchronization of Pulse-Coupled Biological Oscillators, SIAM J. Appl. Math., 50:1645-1662

[47] Mohar, B. (1991) Eigenvalues, Diameter, and Mean Distance in Graphs, Graphs and Combinatorics 7:53-64

[48] Murray, J.D. (1993) Mathematical Biology, Berlin;New York: Springer-Verlag

[49] Nagumo, J., Arimoto, S., and Yoshizawa, S. (1962) An Active Pulse Transmission Line Simulating Nerve Axon, Proc. Inst. Radio Engineers, 50:2061-2070

[50] Neuenschwander, S., Castelo-Branco, M., Baron, J., and Singer, W. (2002) Feed-forward Synchronization: Propagation of Temporal Patterns along the Retinothalamocortical Pathway, Philosophical Transactions: Biological Sciences, 357(1428): 1869-1876

[51] Olfati-Saber, R., and Murray, R.M. (2003) Agreement Problems in Networks with Directed Graphs and Switching Topology, IEEE Conference on Decision and Control

[52] Pecora, L.M., and Carroll, T.L. (1990) Synchronization in Chaotic Systems, Phys. Rev. Lett., 64:821-824

[53] Pecora, L.M., and Carroll, T.L. (March 1998) Master Stability Functions for Synchronized Coupled Systems, Physical Review Letters (March 1998).

[54] Pecora, L.M. (July 1998) Synchronization Conditions and Desynchronizing Patterns in Coupled Limit-cycle and Chaotic Systems, Physical Review E

[55] Pikovsky, A., Rosenblum, M., and Kurths, J. (2003) Synchronization: A Universal Concept in Nonlinear Sciences, Cambridge University Press

[56] Pogromsky, A., Santoboni, G., and Nijmeijer, H. (2002) Partial Synchronization: From Symmetry Towards Stability, Physica D, 172:65-87

[57] Rand, R.H., Holmes, P.J. (1980) Bifurcation of Periodic Motions in Two Weakly Coupled Van der Pol Oscillators, Int. J. Nonlinear Mechanics, 15:387-399

[58] Ravasz, Z.N.E., Vicsek, T., Brechet, T., Barabási, A.L. (June 2000) Physics of The Rhythmic Applause, Physical Review $E$

[59] Reddy, D.V.R., Sen, A., Johnston, G.L. (June 1998) Time Delay Induced Death in Coupled Limit Cycle Oscillators, Physical Review Letters

[60] Reynolds, C. (1987) Flocks, Birds, and Schools: a Distributed Behavioral Model, Computer Graphics, 21:25-34

[61] Rock, I., and Palmer, S. (1990) The Legacy of Gestalt Psychology, Scientific American, 263:84-90

[62] Seiler, P., Pant, A., and Hedrick J.K. (2003) A Systems Interpretation for Observations of Bird V-formations, Journal of Theoretical Biology, 221:279-287

[63] Seung, H.S. (1998) Continuous Attractors and Oculomotor Control, Neural Networks, 11:1253-1258

[64] Singer, W. (1999) Neuronal Synchrony: a Versatile Code for the Definition of Relations, Neuron, 24:49-65

[65] Slotine, J.J.E., and Li, W. (1991) Applied Nonlinear Control, Prentice-Hall

[66] Slotine, J.J.E., and Lohmiller, W. (2001) Modularity, Evolution, and the Binding Problem: A View from Stability Theory, Neural Networks, 14(2)

[67] Slotine, J.J.E. (2003) Modular Stability Tools for Distributed Computation and Control, Int. J. Adaptive Control and Signal Processing, 17(6).

[68] Slotine, J.J.E., and Wang, W. (2003) A Study of Synchronization and Group Cooperation Using Partial Contraction Theory, in S. Morse, N. Leonard, V. Kumar (Editors), Proceedings of Block Island Workshop on Cooperative 
Control, Springer Lecture Notes in Control and Information Science

[69] Smale, S. (1976) A mathematical model of two cells via Turing's equation, in The Hopf Bifurcation and Its Applications, 354-367, Spinger-Verlag, NY

[70] Somers, D.C., and Kopell, N. (1993) Rapid Synchronization Through Fast Threshold Modulation, Biological Cybernetics, 68

[71] Somers, D.C., and Kopell, N. (1995) Waves and Synchrony in Networks of Oscillators of Relaxation and NonRelaxation Type, Physica D

[72] Storti, D.W., Rand, R.H. (1982) Dynamics of Two Strongly Coupled Van der Pol Oscillators, Int. J. Nonlinear Mechanics, 17:143-152

[73] Strogatz, S.H., and Stewart, I. (December 1993) Coupled Oscillators and Biological Synchronization, Scientific American

[74] Strogatz, S.H. (1994) Nonlinear Dynamics and Chaos: with applications to physics, biology, chemistry, and engineering, Addison-Wesley Pub., Reading, MA

[75] Strogatz, S.H. (2000) From Kuramoto to Crawford: exploring the onset of synchronization in populations of coupled oscillators, Physica D: Nonlinear Phenomena, 143(1-4)

[76] Strogatz, S.H. (2001) Exploring Complex Networks, Nature, 410:268-276

[77] Strogatz, S. (2003) Sync: The Emerging Science of Spontaneous Order, New York: Hyperion

[78] Tanner, H., Jadbabaie, A., and Pappas, G.J. (2003) Stable Flocking of Mobile Agents, Part I: Fixed Topology; Part II: Dynamic Topology, IEEE Conference on Decision and Control, Maui, HI

[79] Tanner, H., Jadbabaie, A., and Pappas, G.J. (2003) Coordination of Multiple Autonomous Vehicles, IEEE Mediterranian Conference on Control and Automation, Rhodes, Greece

[80] Turing, A. (1952) The Chemical Basis of Morphogenesis, Philos. Trans. Roy. Soc., B 237

[81] Vicsek, T., Czirok, A., Jacob, E.B., Cohen, I., and Schochet, O. (1995) Novel type of phase transitions in a system of self-driven particles, Physical Review Letters, 75:1226-1229

[82] Wang, W., and Slotine, J.J.E. (2004) A study of continuous attractors and singularly perturbed systems using contraction theory, MIT Nonlinear Systems Laboratory Report 010804

[83] Watts, D.J., and Strogatz, S.H. (1998) Collective Dynamics of 'Small-World' Networks, Nature, 393:440-442

[84] Watts, D.J. (1999) Small Worlds, Princeton University Press

[85] Winfree, A.T. (1967) Biological rhythms and the behavior of populations of coupled oscillators, J. Theor. Biol., 16:15-42

[86] Winfree, A.T. (2000) The Geometry of Biological Time, Springer

[87] Wolf, J.A., Schroeder, L.F., and Finkel, L.H. (July 2001) Computational Modeling of Medium Spiny Projection Neurons in Nucleus Accumbens: Toward the Cellular Mechanisms of Afferent Stream Integration, Proceedings of the IEEE 89(7):1083-1092

[88] Yen, S.C., Menschik E.D., and Finkel, L.H. (June 1999) Perceptual Grouping in Striate Cortical Networks Mediated by Synchronization and Desynchronization, Neurocomputing, 26(7):609-616 